\title{
ALGEBRAIC AND ETALE $K$-THEORY
}

\author{
BY \\ WILLIAM G. DWYER AND ERIC M. FRIEDLANDER ${ }^{1}$
}

\begin{abstract}
We define etale $K$-theory, interpret various conjectures of Quillen and Lichtenbaum in terms of a map from algebraic $K$-theory to etale $K$-theory, and then prove that this map is surjective in many cases of interest.
\end{abstract}

1. Introduction. The purpose of this paper is to study the etale $K$-theory of a commutative noetherian $\mathbf{Z}[1 / l]$-algebra $A$. Etale $K$-theory is relatively easy to compute, and it is related to algebraic $K$-theory by a natural, highly nontrivial map $\phi$. One stimulus for this work has been the conjecture that for many rings $A$ the map $\phi$ is close to an isomorphism; this conjecture is a common generalization of several distinct conjectures made by Lichtenbaum and Quillen $[19,33]$. The main computational result below is Theorem 8.7, which (roughly) states that the map $\phi$ is surjective for suitable subrings of an algebraic number field.

By definition, the etale $K$-theory of $A$ depends upon the etale homotopy type [14] of $\operatorname{Spec} A$ in a very classical way; for instance, there is a spectral sequence of Atiyah-Hirzebruch type (\$5) which relates the continuous etale cohomology of $A$ with certain local coefficients to the etale $K$-theory of $A$. From this point of view, etale $K$-theory is a (twisted) generalized cohomology theory on the etale homotopy type of Spec $A$, a theory which bears the same relationship to etale cohomology as the complex topological $K$-theory of spaces does to singular cohomology. From a pragmatic point of view, the main achievement of etale $K$-theory is to manufacture, for a fairly general ring $A$, some plausible analogue of the space im $J\left(\right.$ or $\left.F \psi^{q}\right)$ used by Quillen to identify the algebraic $K$-theory of a finite field [30]. The present study of etale $K$-theory is an extension to more general rings of the work in [12, 13].

Some of the topological and algebro-geometric material below may be interesting in its own right, for instance, the notion of "geometric function space" $(\S 2)$, the general construction of continuous cohomology ( $\$ 2)$, and the association of a secondary cohomological transfer homomorphism to a finite cyclic covering map (§7). A little more speculative is the hope that, as a new algebraic tool, etale $K$-theory may prove useful in the study of various questions in algebraic geometry which are seemingly unrelated to $K$-theory.

Because of the long gestation period of this paper, there have already been a few publications which make use of the machinery developed here. In collaboration with

Received by the editors December 10, 1984.

1980 Mathematics Subject Classification. Primary 18F25; Secondary 12A60, 55N15.

${ }^{1}$ Both authors were partially supported by a grant from the National Science Foundation. 
V. Snaith and R. Thomason, we showed that etale $K$-theory satisfies "etale cohomological descent" and proved a general surjectivity theorem for a modified form of the map $\phi[8]$. R. Thomason proved that the natural transformation from algebraic to etale $K$-theory satisfies a theorem of Riemann-Roch type [42]; he has also used etale $K$-theory in several other recent papers [41, 43]. In [39], C. Soulé constructed operations in etale $K$-theory.

We acknowledge a great debt to C. Soulé, whose provoctive paper [37] led directly to the present work. Just as important is the pervasive influence below of the ideas of A. K. Bousfield and D. M. Kan (especially [3]).

Outline of the paper. $\$ 2$ contains a technical description of various function spaces and spectral sequences. Many of our constructions are formulated in terms of (topological) spectra rather than of spaces or homotopy groups, and $\$ 3$ describes the categorical machinery needed to produce these spectra. The definition of the etale $K$-theory spectrum and the definition of the map from algebraic to etale $K$-theory appear in $\S 4$, while $\S 5$ contains the specializations to etale $K$-theory of the spectral sequences of $\$ 2$. $\S 6$ discusses the $K$-theory transfer homomorphism, and $\S 7$ an analogous secondary transfer homomorphism for cyclic covering maps. $\S 7$ also contains the proof (by descent) of a surjectivity result for the etale $K$-theory secondary transfer. The surjectivity theorems for rings of $S$-integers in global fields and for fields of $\mathbf{Z} /$ - -cohomological dimension $\leqslant 2$ are proved in $\S 8$, as well as an isomorphism theorem for finite fields and a divisibility theorem for the Borel classes in the algebraic $K$-theory of a ring of integers in a number field. The proofs in $\S 8$ use product, transfer, and secondary transfer to reduce all of the questions to low-degree calculations. The appendix contains a result which is needed in $\$ 4$ in order to recognize a construction of algebraic $K$-theory. An overview of our specific conclusions for algebraic $K$-theory is given in [7].

Notation and terminology. Throughout the paper, $l$ denotes a fixed prime number and $l^{\nu}$ an arbitrary power of $l$. The word spectrum is always used in the sense of algebraic topology [25], and space is a synonym for simplicial set [22]. We will ordinarily make no distinction in notation between a commutative ring $A$ and the associated affine scheme $\operatorname{Spec} A$. All schemes are locally noetherian by assumption; the contraction sscheme then stands for (locally noetherian) simplicial scheme. One background reference for the theory of simplicial schemes is [14], though we have deviated from some of the usages there in the interests of economy. In particular, the word hypercovering is used below to stand for what in [14] is called "rigid hypercovering."

If $A$ and $B$ are objects of some category with a simplicial structure, then $\operatorname{hom}(A, B)$ will denote the set of maps from $A$ to $B$ in that category, and $\operatorname{Hom}(A, B)$ the corresponding space (= simplicial set) of maps from $A$ to $B$. For instance, if $A$ and $B$ are sschemes, then $\operatorname{Hom}(A, B)$ is the simplicial set with $\operatorname{Hom}(A, B)_{n}=\operatorname{hom}(A \otimes \Delta[n], B)$ [14, p. 124]. If $A$ and $B$ are provided with structure maps $A \rightarrow C$ and $B \rightarrow C$ for some $C$, then $\operatorname{hom}(A, B)_{C}$ (respectively $\left.\operatorname{Hom}(A, B)_{C}\right)$ stands for the set (resp. space) of maps from $A$ to $B$ in the category of objects over $C$. 
2. Function spaces and spectral sequences. The purpose of this section is first of all to construct various spaces of functions between pro-spaces and between sschemes. If $S \rightarrow V, T \rightarrow V$ are maps of pro-spaces, there is a space $\operatorname{Hom}_{t}(S, T)$ of topological functions, a space $\operatorname{Hom}_{t}(S, T)_{V}$ of relative topological functions, and a space $\operatorname{Hom}_{l}(S, T)_{V}$ of relative $l$-adic functions. If $X \rightarrow R, Y \rightarrow R$ are maps of sschemes, there is a space $\operatorname{Hom}_{g}(X, Y)_{R}$ of relative geometric functions, as well as a map from $\operatorname{Hom}_{g}(X, Y)_{R}$ to an associated space of $l$-adic functions (see 2.4).

The second part of the section contains the construction of a homotopy spectral sequence associated to $\operatorname{Hom}_{t}(S, T), \operatorname{Hom}_{t}(S, T)_{V}$, or $\operatorname{Hom}_{l}(S, T)_{V}$ (see 2.10). There is a formula for the $E_{2}$-term of this spectral sequence in terms of the "continuous cohomology" (see 2.8 ) of a pro-space with local coefficients.

2.1 Definition. Let $S=\left\{S_{\alpha}\right\}$ and $T=\left\{T_{\beta}\right\}$ be pro-spaces with each $T_{\beta}$ a Kan complex [22, p. 3]. For any $n \geqslant 0$, let $\operatorname{Hom}_{t}^{\langle n\rangle}(S, T)$ denote the space

$$
\underset{\beta}{\operatorname{holim}} \underset{\alpha}{\lim } \operatorname{Hom}\left(S_{\alpha}, T_{\beta}\langle n\rangle\right)
$$

where $T_{\beta}\langle n\rangle$ is the $n$th stage in the simplicial Postnikov tower of $T_{\beta}$ [22, p. 32]. Define the space of topological functions from $S$ to $T$ by

$$
\operatorname{Hom}_{t}(S, T) \equiv \underset{n}{\operatorname{holim}} \operatorname{Hom}_{t}^{\langle n\rangle}(S, T) .
$$

It is clear that $\operatorname{Hom}_{t}(S, T)$ is natural with respect to all maps $S^{\prime} \rightarrow S$ and all strict maps $T \rightarrow T^{\prime}$. (A map of pro-spaces is said to be strict if it is given by a natural transformation with respect to a functor on indexing categories $[14, \S 4]$.) If $S$ and $T$ are singleton pro-spaces (i.e., indexed by the trivial one-object category) then $\operatorname{Hom}_{t}(S, T)$ is homotopy equivalent to the standard function space of maps from $S$ to $T$.

There is also a relative version of (2.1).

2.2 Definition. Let $\left\{T_{\beta} \rightarrow V_{\beta}\right\}$ be a pro-object of Kan fibrations [22, p. 25] and $S=\left\{S_{\alpha}\right\} \rightarrow V=\left\{V_{\beta}\right\}$ some specified map of pro-spaces. For any $n \geqslant 0$, let $\operatorname{Hom}_{t}^{\langle n\rangle}(S, T)_{V}$ denote the space

$$
\underset{\beta}{\operatorname{holim}} \underset{\alpha}{\longrightarrow} \lim \operatorname{Hom}\left(S_{\alpha}, T_{\beta} / V_{\beta}\langle n\rangle\right)_{V_{\beta}},
$$

where $T_{\beta} / V_{\beta}\langle n\rangle$ is the $n$th stage of the simplicial Moore-Postnikov tower of $T_{\beta} \rightarrow V_{\beta}$ [22, p. 34]. Define the space of relative topological functions from $S$ to $T$ over $V$ by

$$
\operatorname{Hom}_{t}(S, T)_{V} \equiv \underset{n}{\operatorname{holim}} \operatorname{Hom}_{t}^{\langle n\rangle}(S, T)_{V} .
$$

It is convenient to use the notation of (2.2) in a slightly larger setting. Let $g$ : $T=\left\{T_{\gamma}\right\}_{\gamma \in \Gamma} \rightarrow V=\left\{V_{\delta}\right\}_{\delta \in \Delta}$ be a strict map determined by a functor $\Phi: \Delta \rightarrow \Gamma$. Construct the associated levelwise fibration $\left\{T_{\beta} \rightarrow V_{\beta}\right\}_{\beta \in B}$ as follows: $B$ is the category in which an object is a triple $(\gamma, \delta, \gamma \rightarrow \Phi(\delta))$ and a map a pair $\left(\gamma^{\prime} \rightarrow \gamma\right.$, $\delta^{\prime} \rightarrow \delta$ ) determining a commutative square; $T_{\beta} \rightarrow V_{\beta}$ is then the mapping fibration of $T_{\gamma} \rightarrow V_{\delta}$ for $\beta=(\gamma, \delta, \gamma \rightarrow \Phi(\delta))$. Any specified map $S \rightarrow V=\left\{V_{\delta}\right\}$ determines a map $S \rightarrow\left\{V_{\beta}\right\}$ and therefore a relative function space $\operatorname{Hom}_{t}\left(S,\left\{T_{\beta}\right\}\right)_{\left\{V_{\beta}\right\}}$. As in Definition 2.2, the notation for this relative function space is $\operatorname{Hom}_{t}(S, T)_{V}$. 
The Bousfield-Kan fibrewise $(\mathbf{Z} / l-)$ completion of a fibration $E \rightarrow B$ appears in one of its forms as a tower of fibrations $\left\{(\mathbf{Z} / l)_{n} E \rightarrow B\right\}_{n \geqslant 0}[3, \mathrm{I}, \S 8]$. If $T=\left\{T_{\gamma}\right\}$ $\rightarrow V=\left\{V_{\delta}\right\}$ is a strict map of pro-spaces, define the fibrewise completion $T_{V} \wedge V$ to be the pro-space $\left\{(\mathbf{Z} / l)_{n}^{\bullet} T_{\beta} \rightarrow V_{\beta}\right\}_{\beta, n}$, where $\left\{T_{\beta} \rightarrow V_{\beta}\right\}$ is the associated levelwise fibration.

2.3 Definition. Let $T \rightarrow V$ be a strict map (of pro-spaces) and $S \rightarrow V$ some specified map. Define the space of relative l-adic functions from $S$ to $T$ over $V$ to be the space

$$
\operatorname{Hom}_{l}(S, T)_{V} \equiv \operatorname{Hom}_{t}\left(S, T_{v}^{\wedge}\right)_{V}
$$

These definitions apply to sschemes through the etale topological type functor, which passes from the category of (locally noetherian) sschemes to the category of pro-spaces and strict maps. If $X$ is a sscheme, its etale topological type $X_{\mathrm{et}}$ is the pro-space $\{\pi \cdot U\}_{U}$ indexed by the hypercoverings $U$ of $X$, where $\pi \cdot U$ is the diagonal of the bi-simplicial set obtained by applying the Zariski connected component functor to each bi-dimension of $U[14, \S 4]$.

2.4 Definition. Let $X \rightarrow R, Y \rightarrow R$ be maps of sschemes. Define the space of relative geometric functions from $X$ to $Y$ over $R$ by

$$
\operatorname{Hom}_{g}(X, Y)_{R} \equiv \underset{U}{\rightarrow} \lim (U, Y)_{R},
$$

where the direct limit is indexed by the hypercoverings $U$ of $X$ and $\operatorname{Hom}(U, Y)_{R}$ signifies $\operatorname{Hom}(\operatorname{diag} U, Y)_{R}$. Define the space of relative l-adic functions from $X$ to $Y$ over $R$ by

$$
\operatorname{Hom}_{l}(X, Y)_{R} \equiv \underset{U}{\rightarrow} \lim _{l}\left(U_{\mathrm{et}}, Y_{\mathrm{et}}\right)_{R_{\mathrm{et}}}
$$

where the direct limit is again indexed by the hypercoverings $U$ of $X$ and $U_{\mathrm{et}}$ denotes the etale topological type of $\operatorname{diag} U$.

If $U$ is a hypercovering of $X$, the natural map $\operatorname{diag} U \rightarrow X$ of sschemes induces a weak equivalence $U_{\mathrm{et}} \rightarrow X_{\mathrm{et}}$ (cf. [14, 8.11]) and therefore a homotopy equivalence $\operatorname{Hom}_{l}\left(X_{\mathrm{et}}, Y_{\mathrm{et}}\right)_{R_{\mathrm{et}}} \rightarrow \operatorname{Hom}_{l}\left(U_{\mathrm{et}}, Y_{\mathrm{et}}\right)_{R_{\mathrm{et}}}$. This indicates that the direct limit in the above definition of $\operatorname{Hom}_{l}(X, Y)_{R}$ has no homotopy function. The purpose of this direct limit is to simplify the following proposition.

2.5 Proposition. Given maps $X \rightarrow R, Y \rightarrow R$ of sschemes, there is a natural map $\operatorname{Hom}_{g}(X, Y)_{R} \rightarrow \operatorname{Hom}_{l}(X, Y)_{R}$.

Proof. For each hypercovering $U$ of $X$ there is a natural map $\operatorname{Hom}(U, Y) \rightarrow$ $\operatorname{Hom}\left(U_{\mathrm{et}}, Y_{\mathrm{et}}\right)$ constructed in [14, 13.2]; it is easy to see that the same procedure gives a map $\operatorname{Hom}(U, Y)_{R} \rightarrow \operatorname{Hom}\left(U_{\mathrm{et}}, Y_{\mathrm{et}}\right)_{R_{\mathrm{ei}}}$. Given any two maps $S \rightarrow V, T \rightarrow V$ of pro-spaces, there is an obvious associated map $\operatorname{Hom}(S, T)_{V} \rightarrow \operatorname{Hom}_{t}(S, T)_{V}$ as well as a map $\operatorname{Hom}_{t}(S, T)_{V} \rightarrow \operatorname{Hom}_{l}(S, T)_{V}$ induced by the fibrewise completion morphism $\left\{T_{\beta} \rightarrow(\mathbf{Z} / l)_{n}^{*} T_{\beta}\right\}_{\beta, n}$. Putting these together produces for each hypercovering $U$ of $X$ a natural map $\operatorname{Hom}(U, Y)_{R} \rightarrow \operatorname{Hom}_{l}\left(U_{\mathrm{et}}, Y_{\mathrm{et}}\right)_{R_{\mathrm{et}}}$. The proof is finished by taking a direct limit over $U$. 
2.6 Remark. Suppose that $X \rightarrow R$ is replaced by a "geometric map" $U \rightarrow R$, where $U$ is a hypercovering of $X$. In this case it is natural to define

$$
\operatorname{Hom}_{g}(X, Y)_{R} \equiv \underset{V}{\lim } \operatorname{Hom}(V, Y)_{R}, \operatorname{Hom}_{l}(X, Y)_{R} \equiv \underset{V}{\lim } \operatorname{Hom}_{l}\left(V_{\mathrm{et}}, Y_{\mathrm{et}}\right)_{R_{\mathrm{et}}},
$$

where $V$ runs through all hypercoverings of $X$ which refine $U$. The construction of Proposition 2.5 again leads to a natural map $\operatorname{Hom}_{g}(X, Y)_{R} \rightarrow \operatorname{Hom}_{l}(X, Y)_{R}$.

The remainder of this section is devoted to the definition of continuous cohomology and the construction of a homotopy spectral sequence associated to $\operatorname{Hom}_{t}(S, T)$ or $\operatorname{Hom}_{t}(S, T)_{V}$.

2.7 Definition. A groupoid $G$ is a small category in which every morphism is invertible. A $G$-module $M$ is a functor from $G$ to the category of abelian groups. A pair $\mathscr{M}=(G, M)$ consisting of a groupoid $G$ and a $G$-module $M$ is called a coefficient system. A morphism $\mathscr{M}=(G, M) \rightarrow \mathscr{M}^{\prime}=\left(G^{\prime}, M^{\prime}\right)$ of coefficient systems is a functor $f: G \rightarrow G^{\prime}$ together with a natural transformation $M^{\prime} \rightarrow M \circ f$. If $\mathscr{M}=(G, M)$, the relative Eilenberg-Mac Lane space $K(\mathscr{M}, n), n \geqslant 0$, is the natural Kan fibration over $N G$ with fibre over $x \in \operatorname{Obj}(G)$ equal to the simplicial EilenbergMac Lane space $K(M(x), n)$ [22, p. 99].

The nerve $N G$ of a groupoid $G$ is the disjoint union of Eilenberg-Mac Lane spaces of the form $K(\pi, 1)$ [34]. Relative Eilenberg-Mac Lane spaces come up naturally in twisted cohomology theory and also in the Postnikov decomposition theory of non-simply-connected spaces [9].

2.8 Definition. Let $S$ be a pro-space, $\mathscr{M}=\left\{\mathscr{M}_{j}\right\}=\left\{\left(G_{j}, M_{j}\right)\right\}$ a pro-object of coefficient systems, and $f: S \rightarrow N G=\left\{N G_{j}\right\}$ a specified map. For each $n \geqslant 0$, define the $n$th continuous cohomology group of $S$ with (local) coefficients $\mathscr{M}$ by

$$
H_{\text {cont }}^{n}(S, \mathscr{M}) \equiv \pi_{0} \operatorname{Hom}_{t}(S, K(\mathscr{M}, n))_{N G} .
$$

If $\mathscr{M}=(G, M)$ is a constant pro-object and $S=\left\{S_{\alpha}\right\}$, then

$$
\begin{aligned}
H_{\text {cont }}^{n}(S, \mathscr{M}) & =\pi_{0} \underset{\underset{\alpha}{\lim }}{\rightarrow} \operatorname{Hom}\left(S_{\alpha}, K(\mathscr{M}, n)\right)_{N G} \\
& =\underset{\rightarrow}{\lim } H^{n}\left(S_{\alpha}, \mathscr{M}\right)=H^{n}(S, \mathscr{M}),
\end{aligned}
$$

where $H^{n}(S, \mathscr{M})$ and $H^{n}\left(S_{\alpha}, \mathscr{M}\right)$ denote the usual local cohomology groups of $S$ or $S_{\alpha}$ with respect to the coefficient system $\mathscr{M}$ and the twisting maps $S_{\alpha} \rightarrow N G$. For a general $\mathscr{M}=\left\{\mathscr{M}_{j}\right\}_{j \in J}$, there is a more complicated relationship between continuous cohomology with coefficients $\mathscr{M}$ and ordinary cohomology:

2.9 Proposition (CF. [40, 2.2]). With the above notation, there is a convergent first quadrant spectral sequence

$$
E_{2}^{p, q}=\lim _{J}^{p} H^{q}\left(S, \mathscr{M}_{j}\right) \Rightarrow H_{\mathrm{cont}}^{p+q}(S, \mathscr{M})
$$

in which the rth differential $d_{r}$ has bi-degree $(r, 1-r)$. 
REMARK. Here $\lim _{J}^{p}$ is the $p$ th right derived functor of the inverse limit functor on $J$-diagrams of abelian groups [3, XI, §6].

Proof. Pick $n \geqslant 1$ and apply $\operatorname{Hom}_{t}(S,-)_{N G}$ to the homotopy fibre square:

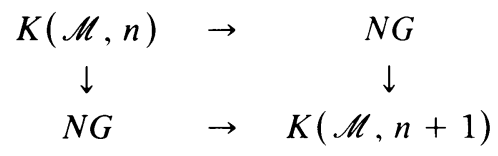

What results is a homotopy fibre square of spaces in which two of the corners are contractible - this leads to the conclusion that $\operatorname{Hom}_{t}(S, K(\mathscr{M}, n))_{N G}$ is homotopy equivalent to the loop space $\Omega \operatorname{Hom}_{t}(S, K(\mathscr{M}, n+1))_{N G}$. It follows by induction that for any $n \geqslant 0$ there are natural isomorphisms

$$
\pi_{q} \operatorname{Hom}_{t}(S, K(\mathscr{M}, n))_{N G} \approx H_{\text {cont }}^{n-q}(S, \mathscr{M}) .
$$

In light of this calculation, the Bousfield-Kan spectral sequence [3, XI, §7] associated to the homotopy inverse limit formula

$$
\operatorname{Hom}_{t}(S, K(\mathscr{M}, n))_{N G}=\underset{J}{\operatorname{holim}} \underset{\alpha}{\lim } \operatorname{Hom}\left(S_{\alpha}, K\left(\mathscr{M}_{j}, n\right)\right)_{N G}
$$

can be re-indexed to give a (suitably fringed) first quadrant spectral sequence

$$
E_{\alpha}^{p, q}(n)= \begin{cases}\lim _{J}{ }^{p} H^{q}\left(S_{\alpha}, \mathscr{M}_{j}\right), & p+q \leqslant n, \\ 0 & p+q>n,\end{cases}
$$

which converges to $H_{\text {cont }}^{p+q}(S, \mathscr{M})$ for $p+q<n$. It is not hard to produce for each $n$ a map $E_{r}^{p, q}(n+1) \rightarrow E_{r}^{p, q}(n)$ which in total degree $\leqslant n$ gives an isomorphism on $E_{2}$-terms. The spectral sequence of the proposition is then $\lim _{n} E_{r}^{r, q}(n)$.

If $T_{\beta}$ is a space; let $\Gamma T_{\beta}$ denote the fundamental groupoid of $T_{\beta}$ and for $n>1$ (and $n=1$ if $\pi_{1}$ is abelian), let $\pi_{n}\left(T_{\beta},-\right)$ be the $\Gamma T_{\beta}$-module whose value on a vertex $\alpha$ of $T_{\beta}$ is $\pi_{n}\left(T_{\beta}, \alpha\right)$. Let $\Pi_{n} T_{\beta}$ be the coefficient system $\left\{\Gamma T_{\beta}, \pi_{n}\left(T_{\beta},-\right)\right\}$ and, finally, if $T=\left\{T_{\beta}\right\}$ is a pro-space, let $\Pi_{n}(T)$ denote $\left\{\Pi_{n} T_{\beta}\right\}$.

2.10 Proposition. Let $f: S \rightarrow T=\left\{T_{\beta}\right\}$ be a map of pro-spaces in which each $T_{\beta}$ is a connected Kan complex with abelian fundamental group. Then there is a natural fourth quadrant spectral sequence

$$
E_{2}^{p, q}=H_{\text {cont }}^{p}\left(S, \Pi_{-q} T\right) \Rightarrow \pi_{-(p+q)}\left(\operatorname{Hom}_{t}(S, T), f\right)
$$

in which the differential $d_{r}$ has bi-degree $(r, 1-r)$. This spectral sequence converges completely in positive degrees if $\lim _{\leftarrow}{ }_{r}^{1} E_{r}^{p, q}$ vanishes for all $p \geqslant 0, p+q \geqslant 0$.

Proof. This spectral sequence is a re-indexed form of the one associated by [3, IX, 4.2] to the tower of fibrations $\left\{\operatorname{Hom}_{t}^{\langle n\rangle}(S, T)\right\}_{n \geqslant 0}$. Let $S=\left\{S_{\alpha}\right\}$. It follows from the functorial approach to obstruction theory [9] that there is a natural homotopy fibre sequence

$$
\operatorname{Hom}\left(S_{\alpha}, K\left(\Pi_{n} T_{\beta}, n\right)\right)_{N \Gamma T_{\beta}} \rightarrow \operatorname{Hom}\left(S_{\alpha}, T_{\beta}\langle n\rangle\right) \rightarrow \operatorname{Hom}\left(S_{\alpha}, T_{\beta}\langle n-1\rangle\right)
$$

for any $\alpha, \beta$ and any $n>0$. This determines fibre sequences

$$
\operatorname{Hom}_{t}\left(S, K\left(\Pi_{n} T, n\right)\right)_{N \Gamma T} \rightarrow \operatorname{Hom}_{t}^{\langle n\rangle}(S, T) \rightarrow \operatorname{Hom}_{t}^{\langle n-1\rangle}(S, T) .
$$


The identification of

$$
E_{2}^{p, q}=\pi_{-(p+q)} \operatorname{Hom}_{t}\left(S, K\left(\Pi_{-q} T,-q\right)\right)_{N \Gamma T}
$$

with $H_{\text {cont }}^{p}\left(S, \Pi_{-q} T\right)$ is a consequence of the initial remarks in the proof of Proposition 2.9. The convergence criterion is that of [3, IX, 5.4].

There is also a relative version of the spectral sequence. Suppose that $g_{\beta}: T_{\beta}$ $\rightarrow V_{\beta}$ is a fibration which has a connected fibre with abelian fundamental group. Denote by $\pi_{n}\left(T_{\beta} / V_{\beta},-\right)$ the $\Gamma T_{\beta}$-module which assigns to each vertex $x$ of $T_{\beta}$ the group $\pi_{n}\left(g_{\beta}^{-1} g_{\beta}(x), x\right)$, and by $\Pi_{n} T_{\beta} / V_{\beta}$ the corresponding coefficient system $\left(\Gamma T_{\beta}, \pi_{n}\left(T_{\beta} / V_{\beta},-\right)\right)$.

2.11 Proposition. Let $\left\{g_{\beta}: T_{\beta} \rightarrow V_{\beta}\right\}$ be a pro-object of fibrations each of which has connected fibre with abelian fundamental group, and let $f=S \rightarrow T$ be a map. Then there is a natural fourth quadrant spectral sequence

$$
E_{2}^{p, q}=H_{\text {cont }}^{p}\left(S, \Pi_{-q} T / V\right) \Rightarrow \pi_{-(p+q)}\left(\operatorname{Hom}_{t}(S, T)_{V}, f\right)
$$

in which the differential $d_{r}$ has bi-degree $(r, 1-r)$. (Here $\Pi_{-q} T / V$ denotes $\left\{\Pi_{-q} T_{\beta} / V_{\beta}\right\}$.) This spectral sequence converges completely in positive degrees if $\lim _{r}^{1} E_{r}^{p, q}$ vanishes for all $p \geqslant 0, p+q \geqslant 0$.

Proof. This is identical to the proof of Proposition 2.10, except that it uses relative obstruction theory fibration sequences

$$
\begin{aligned}
\operatorname{Hom}\left(S_{\alpha}, K\left(\Pi_{n} T_{\beta} / V_{\beta}, n\right)\right)_{N \Gamma T_{\beta}} & \rightarrow \operatorname{Hom}\left(S_{\alpha}, T_{\beta} / V_{\beta}\langle n\rangle\right)_{V_{\beta}} \\
& \rightarrow \operatorname{Hom}\left(S_{\alpha}, T_{\beta} / V_{\beta}\langle n-1\rangle\right)_{V_{\beta}} \quad\left(\text { where } S=\left\{S_{\alpha}\right\}\right) .
\end{aligned}
$$

3. Categorical constructions of spectra. One way to construct a spectrum [35] is to pass from a permutative category to an $\mathscr{F}$-space (see 3.3$)$ and then from the $\mathscr{F}$-space to a spectrum (see 3.2). This section sketches that procedure and then recapitulates the first step with all of the spaces involved replaced by sschemes. For instance, Proposition 3.4 associates an $\mathscr{F}$-sscheme to a particular permutative-categorysscheme made up of general linear groups. $\S 4$ will combine this $\mathscr{F}$-sscheme with the function space constructions of $\S 2$ to obtain $K$-theory $\mathscr{F}$-spaces and $K$-theory spectra.

3.1 Definition [35]. Let $\mathscr{F}$ be the category of finite pointed sets together with basepoint-preserving maps and let $\mathbf{n}$ denote the set $\{0,1, \ldots, n\}$ pointed by 0 . If $\mathscr{C}$ is any category, then an $\mathscr{F}$-object of $\mathscr{C}$ is a functor $\Phi: \mathscr{F} \rightarrow \mathscr{C}$; such a functor amounts to a collection of objects $\Phi(\mathbf{n}), n \geqslant 0$, together with a map $\Phi(\mathbf{n}) \rightarrow \Phi(\mathbf{m})$ for each pointed map $\mathbf{n} \rightarrow \mathbf{m}$. An $\mathscr{F}$-object in the category of spaces is called an $\mathscr{F}$-space. The $\mathscr{F}$-space $\Phi$ is special if the natural map $\Phi(A \vee B) \rightarrow \Phi(A) \times \Phi(B)$ is an equivalence for every pair of finite pointed sets $A$ and $B$.

3.2 Proposition $[35,24,25]$. There is a natural functor which associates to each $\mathscr{F}$-space $\Phi$ an $\Omega$-spectrum $\operatorname{Sp}(\Phi)$. If $\Phi$ is special, then the zeroth space $\operatorname{Sp}(\Phi)_{0}$ of $\operatorname{Sp}(\Phi)$ has the homotopy type of a group completion $[23,26]$ of $\Phi(\mathbf{1})$. 
If $\Phi$ is special, the folding map $1 \vee 1 \rightarrow 1$ determines the $H$-space structure on $\Phi(1)$ which figures in the above group completion. The pre-spectrum which gives rise to $\operatorname{Sp}(\Phi)$ is determined by the canonical maps $S^{1} \wedge \Phi\left(S^{n}\right) \rightarrow \Phi\left(S^{n+1}\right)$, where $S^{1}$ is the standard simplicial model of the circle, $S^{n}$ is the $n$-fold smash power of $S^{1}$, and $\Phi\left(S^{n}\right)$ is the diagonal of the bi-simplicial set obtained by applying $\Phi$ to each dimension of $S^{n}$.

$A$ permutative category is a small category $P$ together with a strictly unital, strictly associative, coherently commutative "sum" operation, denoted $\square$. A morphism between two permutative categories is a functor which strictly respects both the units and the appropriate sum constructions [24, Definition 1]. The nerve [34] of a category $P$ is denoted $N P$.

3.3 Proposition [35; 24, CONST. 10]. There is a natural way of passing from a permutative category $P$ to an $\mathscr{F}$-object $\bar{P}$ of permutative categories, with $\bar{P}(\mathbf{1})=P$. The associated $\mathscr{F}$-space $N \bar{P}$ is special, and $\operatorname{Sp}(N \bar{P})_{0}$ has the homotopy type of $\Omega B(N P)$, where the classifying space $B(N P)$ is formed by using the monoid structure on NP induced by the sum operation of $P$.

The categories $\bar{P}(\mathbf{n}), n \geqslant 0$, are defined as follows: An object of $\bar{P}(\mathbf{n})$ consists of an object $P_{S}$ of $P$ for each subset $S \leqslant \mathbf{n}$ containing 0 (with $P_{\{0\}}$ the unit object of $P$ ) together with compatible isomorphisms $P_{S \cup T} \rightarrow P_{S} \square P_{T}$ whenever $S \cap T=\{0\}$; a morphism of $\bar{P}(\mathbf{n})$ consists of a collection of maps $f_{S}: P_{S} \rightarrow P_{S}^{\prime}$ (with $f_{\{0\}}$ the identity) such that each of the following diagrams commute:

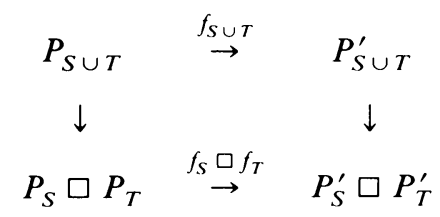

It is not hard to see that $P(\mathbf{n})$ is categorically equivalent to (but not in general isomorphic to) the $n$-fold cartesian power of $P$, and that $N P(\mathbf{n})$ therefore contains $N P \times \cdots \times N P$ as a simplicial deformation retract [24].

Fix a sscheme $R$, and let $\mathscr{C}$ be the category sschemes $/ R$ of sschemes over $R$. For each $n \geqslant 0$ the general linear group sscheme $\mathrm{GL}_{n}\left(\equiv \mathrm{GL}_{n, \mathrm{Z}} \times{ }_{\mathrm{Z}} R\right)$ gives rise in the spirit of [21, p. 75] to a category in $\mathscr{C}$ with object "set" the sscheme $\mathrm{GL}_{n}$, and composition law the matrix multiplication map $\mathrm{GL}_{n} \times{ }_{R} \mathrm{GL}_{n} \rightarrow \mathrm{GL}_{n}$. This category in $\mathscr{C}$ is denoted $\mathscr{G} \ell_{n}$.

3.4 Proposition. The disjoint union $\amalg_{k \geqslant 0} \mathscr{G}_{k} \equiv \mathscr{G} \ell_{*}$ has the structure of a permutative category in $\mathscr{C}$ in which the sum operation $\square$ is determined by the external sum maps

$$
\mathrm{GL}_{m} \times{ }_{R} \mathrm{GL}_{n} \rightarrow \mathrm{GL}_{m+n}
$$

The proof is immediate.

3.5 Proposition $[11,9.1]$. Let $R$ be a sscheme and $\mathscr{G} \ell_{*}$ the permutative category in sschemes $/ R$ described in Proposition 3.4. Then there is a naturally associated $\mathscr{F}$-object

$$
B \overline{\mathscr{G} \ell_{*}}: F \rightarrow \text { sschemes } / R
$$


such that

$$
B \overline{\mathscr{G} \ell_{*}}(\mathbf{0})=R, \quad B \overline{\mathscr{G} \ell_{*}}(\mathbf{1})=\coprod_{k \geqslant 0} B \mathrm{GL}_{k},
$$

and $\overline{B \bar{G} \ell_{*}}(\mathbf{n})$ contains the $n$-fold fibre power of $\overline{B \mathscr{G} \ell_{*}}(\mathbf{1})$ over $R$ as a simplicial deformation retract.

In the above proposition, $B \mathrm{GL}_{k}$ is the diagonal of the bi-simplicial sscheme $N \mathrm{GL}_{k}$ $[14,1.2, \S 2]$. The construction of $\overline{B \mathscr{G} \ell_{*}}$ is carried out by formally imitating the construction of $N \bar{P}$ in (3.3). This involves first building a functor,

$$
\mathscr{G}_{*}: \mathscr{F} \rightarrow \text { (permutative categories in sschemes } / R \text { ) }
$$

and then obtaining $\overline{B \mathscr{G} \ell_{*}}(\mathbf{n})$ as the diagonal of the nerve of $\overline{\mathscr{G} \ell}{ }_{*}(\mathbf{n})$. (See $[\mathbf{1 4}, 5.2$, $8.3,9.1$ ] for more details.)

The following pairing on $B \overline{\mathscr{G} \ell}$ * will induce a product structure on $K$-theory. Let $\Lambda: \mathscr{F} \times \mathscr{F} \rightarrow \mathscr{F}$ denote the smash product functor on pointed sets sending $(\mathbf{m}, \mathbf{n})$ to mn [25, p. 303].

3.6 Proposition $[13,1.4]$. The $\mathscr{F}$-object $B \overline{G \mathscr{G}}{ }_{*}$ of $(3.5)$ admits a natural pairing derived from the external tensor product maps $\mathrm{GL}_{m} \times_{R} \mathrm{GL}_{n} \rightarrow \mathrm{GL}_{m n}$. In particular, there is a functor $\tilde{B} \overline{G \ell_{*}}: \mathscr{F} \times \mathscr{F} \rightarrow$ sschemes $/ R$ together with natural transformations

$$
B \overline{\mathscr{G} \ell_{*}} \circ \Lambda \stackrel{f}{\leftarrow} \tilde{B} \overline{\mathscr{G} \ell_{*}} \stackrel{g}{\rightarrow} B \overline{\mathscr{G} \ell_{*}} \times{ }_{R} B \overline{\mathscr{G} \ell_{*}}
$$

such that for any $(\mathbf{m}, \mathbf{n})$ in $\mathscr{F} \times \mathscr{F}$ the induced map

$$
g_{(\mathbf{m}, \mathbf{n})}: \tilde{B} \overline{\mathscr{G} \ell_{*}}(\mathbf{m}, \mathbf{n}) \rightarrow B \overline{\mathscr{G} \ell_{*}}(\mathbf{m}) \times_{R} B \overline{\mathscr{G} \ell_{*}}(\mathbf{n})
$$

is a simplicial equivalence. In the appropriate sense [25, p. 339] this pairing is associative and commutative.

The assertion that $g_{(\mathbf{m}, \mathbf{n})}$ is a simplicial equivalence means in this case that the map has a right inverse $r_{(\mathbf{m}, \mathbf{n})}$ such that the composite $r_{(\mathbf{m}, \mathbf{n})}{ }^{\circ} g_{(\mathbf{m}, \mathbf{n})}$ is simplicially homotopic to the identity as a map of simplicial schemes over $R$. The above pairing is obtained by reproducing the constructions of [25, Appendix] in the algebraic category.

4. Algebraic and etale $K$-theory. From this point on in the paper, $R$ will denote the ring $\mathbf{Z}[1 / l]$. In this section we will define the algebraic $K$-theory of an affine scheme over $R$ (see 4.1) as well as the etale $K$-theory of a general sscheme over $R$ (see 4.3); the fundamental similarity between these two definitions provides a natural map $\phi$ from algebraic to etale $K$-theory (see 4.4 ). Proposition 4.2 verifies that the algebraic $K$-theory of Definition 4.1 agrees with Quillen $K$-theory [32], while Proposition 4.5 shows that in most cases etale $K$-theory has a straightforward homotopy-theoretic interpretation. The last part of the section deals with properties of the natural transformation $\phi$ in degrees 0 and 1 .

Here and in what follows, $B \overline{\mathscr{G} \ell} *$ is the $\mathscr{F}$-sscheme over $R$ defined in Proposition 3.5 and $\tilde{B} \overline{\mathscr{G} \ell}{ }_{*}$ the associated $\mathscr{F} \times \mathscr{F}$-sscheme of Proposition 3.6. The $\bmod l^{\nu}$ Moore spectrum $S^{0} \cup_{l^{\nu}} e^{1}$ with bottom cell in stable dimension zero is denoted $M(\nu)$. 
4.1 Definition. For any $R$-algebra $A$, the algebraic $K$-theory spectrum of $A$ is the ring spectrum

$$
\mathbf{K}_{A}=\operatorname{Sp}\left(\operatorname{Hom}_{g}\left(A, B \overline{\mathscr{G} \ell_{*}}\right)_{R}\right)
$$

with ring structure provided as in [25, p. 339] by the $\mathscr{F} \times \mathscr{F}$-space $\operatorname{Hom}_{g}\left(A, \tilde{B} \overline{\mathscr{G} \ell_{*}}\right)_{R}$ and tine associated maps involving it (see Proposition 3.6). The algebraic $K$-theory space $K_{A}$ is the ring space $K_{A}=\left(\mathbf{K}_{A}\right)_{0}$. The algebraic $K$-groups of $A$ are defined for $i \geqslant 0$ by

$$
K_{i}(A)=\pi_{i} \mathbf{K}_{A}, \quad K_{i}\left(A, \mathbf{Z} / l^{\nu}\right)=\pi_{i}\left(\mathbf{K}_{A} \wedge \mathscr{M}(\nu)\right) .
$$

REMARK. To obtain the above ring structure, [25] requires that the natural map (see 3.6).

$$
\operatorname{Hom}_{g}\left(A, \tilde{B} \overline{\mathscr{G} \ell_{*}}\right)_{R} \rightarrow \operatorname{Hom}_{g}\left(A, B \overline{\mathscr{G} \ell_{*}}\right)_{R} \times \operatorname{Hom}_{g}\left(A, B \overline{\mathscr{G} \ell_{*}}\right)_{R}
$$

be an equivalence. This follows from Proposition 3.6 and the fact that a simplicial homotopy $Y \otimes \Delta[1] \rightarrow Z$ induces a homotopy $\operatorname{Hom}_{g}(X, Y) \times \Delta[1] \rightarrow \operatorname{Hom}_{g}(X, Z)$.

4.2 Proposition. For any $R$-algebra $A$, the ring $\pi_{*} \mathbf{K}_{A}=\pi_{*}\left(K_{A}\right)$ is naturally isomorphic to the Quillen $K$-theory of $A$ [32] with its usual ring structure [20].

Proof. By Proposition 3.2, $K_{A}$ is a group completion of the space

$$
\coprod_{n \geqslant 0} \operatorname{Hom}_{g}\left(A, B \mathrm{GL}_{n}\right)_{R}=\operatorname{Hom}_{g}\left(A, B \overline{\mathscr{G} \ell_{*}}\right)_{R}(\mathbf{1})
$$

with respect to the external direct sum operation. By results in the appendix, this union of geometric function spaces is equivalent (in a way which preserves the sum operation) to $\amalg_{P} B$ Iso $(P)$, where $P$ runs through the set of isomorphism classes of vector bundles over $A$ (= finitely generated projective $A$-modules). The first part of the proposition now follows from [16, p. 228 and 26]. A crucial point [16, p. 226] is that every epimorphism of vector bundles over $A$ can be split-this is false in general for a nonaffine scheme. The statement about products follows as in $[25, \mathrm{p}$. 302].

4.3 Definition. For any (locally noetherian) sscheme $X$ over $R$, the etale $K$-theory spectrum of $X$ is the ring spectrum

$$
\hat{K}_{X}^{\mathrm{et}}=\operatorname{Sp}\left(\operatorname{Hom}_{l}\left(X, B \overline{\mathscr{G} \ell_{*}}\right)_{R}\right)
$$

with ring structure provided as in $\left[25\right.$, p. 339] by the $\mathscr{F} \times \mathscr{F}$ space $\operatorname{Hom}_{l}\left(X, \tilde{B} \overline{\mathscr{G} \ell_{*}}\right)_{R}$ and the associated maps involving it (see Proposition 3.6). The etale $K$-theory space $\hat{K}_{X}^{\mathrm{et}}$ is the ring space $\hat{K}_{X}^{\mathrm{et}}=\left(\hat{\mathbf{K}}_{X}^{\mathrm{et}}\right)_{0}$. The etale $K$-groups of $X$ are defined for $i \geqslant 0$ by

$$
\hat{K}_{i}^{\mathrm{et}}(X)=\pi_{i} \hat{\mathbf{K}}_{X}^{\mathrm{et}}, \quad \hat{K}_{i}^{\mathrm{et}}\left(X, \mathbf{Z} / l^{\nu}\right)=\pi_{i}\left(\hat{\mathbf{K}}_{X}^{\mathrm{et}} \wedge \mathscr{M}(\nu)\right) .
$$

4.4 Proposition. For any noetherian R-algebra $A$, the map of Proposition 2.5 determines a natural map of ring spectra $\phi: \mathbf{K}_{A} \rightarrow \hat{\mathbf{K}}_{A}^{\mathrm{et}}$. This map induces homomorphisms

$$
\phi_{*}: K_{*}(A) \rightarrow \hat{K}_{*}^{\mathrm{ct}}(A), \quad \phi_{*}: K_{*}\left(A, \mathbf{Z} / l^{\nu}\right) \rightarrow K_{*}^{\mathrm{ct}}\left(A, \mathbf{Z} / l^{\nu}\right),
$$

which are ring homomorphisms for $l^{\nu} \neq 2$. Moreover, the rings involved are associative for $l^{\nu} \neq 3$ and commutative for $l^{\nu} \neq 4,8$. 
Proof. This follows immediately from the naturality of (2.5), the naturality properties of the pairing construction in [25], and the results of [1].

REMARK. The natural transformation (4.4) can be extended from neotherian $R$-algebras to schemes quasi-projective over such algebras by "Jouanalou's device" [13, proof of 1.3]. However, this construction has the disadvantage of being natural only up to homotopy.

Assuming finite cohomological dimension, we give a more explicit formula for the etale $K$-space.

4.5 Proposition. Let $X$ be a connected sscheme over $R$ with (etale) $\mathbf{Z} /$ l-cohomological-dimension [28, VI.1] equal to $d<\infty$. Then for any $n \geqslant 1$ there is a natural homotopy class of maps $\mathbf{Z} \times \operatorname{Hom}_{l}\left(X, B \mathrm{GL}_{n}\right)_{R} \rightarrow \hat{K}_{X}^{\text {et }}$ which is a $(2 n-d)$ equivalence. In addition, for any $n \geqslant 1$ there exists a natural homotopy class of maps

$$
\left(\mathbf{K}_{X}^{\mathrm{et}}\right)_{n} \rightarrow \operatorname{Hom}_{l}\left(X, B \overline{\mathscr{G \ell}_{*}}\left(S^{n}\right)\right)_{R}
$$

such that the induced map

$$
\hat{K}_{X}^{\mathrm{et}} \rightarrow \Omega^{n} \operatorname{Hom}_{l}\left(X, B \overline{\mathscr{G} \ell_{*}}\left(S^{n}\right)\right)_{R}
$$

is a weak equivalence on the connected component of the base point.

Proof. Recall from [14, §8] that the homotopy fibre of $B \overline{\mathscr{G} \ell_{*}}(n)_{R} \rightarrow R_{\mathrm{et}}$ is equivalent to $\left[\left(\amalg_{k \geqslant 0} B G L_{k}(\mathbf{C})\right)^{\times n}\right]^{\wedge}$, where $B \mathrm{GL}_{k}(\mathbf{C})$ is the classifying space of the Lie group $\mathrm{GL}_{k}(\mathbf{C})$. It follows that $\operatorname{Hom}_{l}\left(X, \overline{B \mathscr{G} \ell_{*}}\right)_{R}$ is a special $\mathscr{F}_{\text {-space. Since }}$ $\left(B \mathrm{GL}_{n}\right)_{R}^{\wedge} \rightarrow\left(B \mathrm{GL}_{n+1}\right)_{R}$ is a fibrewise $(2 n+1)$-equivalence for each $n \geqslant 0$, the induced map

$$
\operatorname{Hom}_{l}\left(X, B \mathrm{GL}_{n}\right)_{R} \rightarrow \operatorname{Hom}_{l}\left(X, B \mathrm{GL}_{n+1}\right)_{R}
$$

is a $(2 n-d)$-equivalence (see the proof of 2.10 for the obstruction theory that leads to this conclusion). Therefore $\hat{K}_{X}^{\mathrm{et}}$, as a group completion of

$$
\operatorname{Hom}_{l}\left(X, B \overline{\mathscr{G} \ell_{*}}(\mathbf{1})\right)_{R}=\coprod_{k \geqslant 0} \operatorname{Hom}_{l}\left(X, B \mathrm{GL}_{k}\right)_{R} \text {, }
$$

is equivalent to $\mathbf{Z} \times \lim _{k} \operatorname{Hom}_{l}\left(X, B \mathrm{GL}_{k}\right)_{R}$ [26]. The first part of the lemma follows.

If $S$ is a space and $T_{*}$ a simplicial space, there is a natural map

$$
\operatorname{diag} \operatorname{Hom}\left(S, T_{*}\right) \rightarrow \operatorname{Hom}\left(S, \operatorname{diag} T_{*}\right)
$$

which sends a $k$-simplex $S \times \Delta[k] \rightarrow T_{k}$ to the composite $S \times \Delta[k] \rightarrow T_{k} \times \Delta[k]$ $\rightarrow \operatorname{diag} T_{*}$. Since $\overline{B \mathscr{G} \ell_{*}}\left(S^{n}\right)_{R}^{\wedge}$ receives a map over $R_{\text {et }}$ from the diagonal of $k \mapsto$ $B \overline{\mathscr{G} \ell_{*}}\left(\left(S^{n}\right)_{k}\right)_{R}$, this construction determines a map $\left(\hat{\mathbf{K}}_{X}^{\mathrm{et}}\right)_{n} \rightarrow$ $\operatorname{Hom}_{l}\left(X, B \bar{G} \ell_{*}\left(S^{n}\right)\right)_{R}$. Looping $n$ times produces the stated equivalence because the natural map

$$
\Sigma^{n} / R \wedge\left(\mathbf{Z} \times \underset{\vec{k}}{\lim }\left(B \mathrm{GL}_{k}\right)_{R}^{\wedge}\right) \rightarrow B \overline{\mathscr{G} \ell_{*}}\left(S^{n}\right)_{R}^{\wedge}
$$

has an adjoint which is a weak equivalence on each connected component. (Here $\Sigma^{n} / R \wedge(-)$ is the $n$-fold fibrewise suspension functor over $R_{\mathrm{ct}}$.) 
In [13] the groups $\hat{K}_{i}^{\mathrm{et}}(X)$ and $K_{i}^{\mathrm{et}}\left(X, \mathbf{Z} / l^{\nu}\right)$ are defined in terms of the homotopy groups of the space $\lim _{n} \operatorname{Hom}_{l}\left(X, B \mathrm{GL}_{n, F}\right)$, where $X$ is a sscheme defined over a complete discrete valuation ring $F$ over $R$ with separably closed residue field and $B \mathrm{GL}_{n, F}=B \mathrm{GL}_{n} \times_{R} F$.

4.6 CoRollary. If $X$ is as above and has finite $\mathbf{Z} /$-cohomological dimension, the etale $K$-groups $\hat{K}_{i}^{\mathrm{et}}(X)$ with $i>0$ and $K_{j}^{\mathrm{et}}\left(X, \mathbf{Z} / l^{\nu}\right)$ are isomorphic to those defined in [13].

Proof. For $\hat{K}_{i}^{\text {et }}(X)$ with $i>0$ and $K_{j}^{\text {et }}\left(X, \mathbf{Z} / l^{\nu}\right)$ with $j>1$, it suffices by Proposition 4.5 to prove that the natural maps

$$
\operatorname{Hom}_{l}\left(X, B \mathrm{GL}_{n, F}\right) \rightarrow \operatorname{Hom}_{l}\left(X, B \mathrm{GL}_{n}\right)_{R}
$$

are equivalences for each $n \geqslant 0$. Since $F_{\text {et }}$ is contractible and since the structure map $X_{\text {et }} \rightarrow R_{\text {et }}$ factors through $F_{\text {et }}$, these equivalences are implies by the fibration sequence $\left(B \mathrm{GL}_{n, F}\right)^{\wedge} \rightarrow\left(B \mathrm{GL}_{n}\right) \hat{R} \rightarrow R_{\mathrm{et}}$. For $j=0$ or 1, apply the argument of [13, $1.2]$ in conjunction with the above equivalences.

Let Grass $_{m, n}$ denote the projective $R$-scheme representing locally free coherent sheaves of rank $n$ generated by $m$ global sections, According to [10] there are natural maps (in the category of sschemes over $R$ )

$$
\text { Grass }_{m, n} \leftarrow B\left(\mathrm{GL}_{m} / \mathrm{GL}_{m-n}, \mathrm{GL}_{n, *}\right) \rightarrow B \mathrm{GL}_{n}
$$

such that on $l$-adic etale topological types the left-hand map induces a fibrewise equivalence over $R_{\mathrm{et}}$ and the right-hand map a fibrewise $(2 m-2 n+1)$-equivalence.

4.7 Proposition. If $X$ is a connected sscheme over $R$ of finite $\mathbf{Z} /$-cohomological dimension, there is a natural isomorphism

$$
\theta_{0}: \hat{K}_{0}^{\text {et }}(X) \rightarrow \mathbf{Z} \times \underset{\rightarrow}{\lim } \underset{\vec{k}}{\lim _{0}} \pi_{0} \operatorname{Hom}_{l}\left(X, \text { Grass }_{n+k, n}\right)_{R} .
$$

Moreover, if $X=\operatorname{Spec} A$ is affine and $P$ is a rank $n$ projective $A$-module, then $\theta_{0} \phi([P])$ is represented by the pair $\left(n, \tau_{P}^{\wedge}: A_{\mathrm{et}} \rightarrow\left(\right.\right.$ Grass $\left.\left._{m, n}\right) \hat{R}\right)$ where $\tau_{P}: A \rightarrow$ Grass $_{m, n}$ is the classifying map associated to some surjection $A^{\oplus m} \rightarrow P$.

Proof. The existence of $\theta_{0}$ follows from Proposition 4.5 and the above remarks. For the second statement, choose a splitting $A^{\oplus m} \simeq P \oplus Q$ for some surjection $A^{\oplus m} \rightarrow P$. If $U \rightarrow A$ is an etale hypercovering such that the restriction of $P$ to $U_{0}$ is trivial, then a choice of trivialization for $P$ on $U_{0}$ determines $g_{P}: U \rightarrow B \mathrm{GL}_{n}$ (as in the appendix) while the splitting $A^{\oplus m}=P \oplus Q$ determines a lift $\tilde{g}_{P}: U \rightarrow$ $B\left(\mathrm{GL}_{m} / \mathrm{GL}_{m-n}, \mathrm{GL}_{n, *}\right)$. By construction, $\phi([P])$ is represented by the homotopy class $g_{P} \hat{P}: U_{\text {et }} \rightarrow\left(B \mathrm{GL}_{n}\right)_{R}$ in $\pi_{0} \operatorname{Hom}_{l}\left(U, B \mathrm{GL}_{n}\right)_{R}$, so to prove the proposition it is enough to check that the following square commutes:

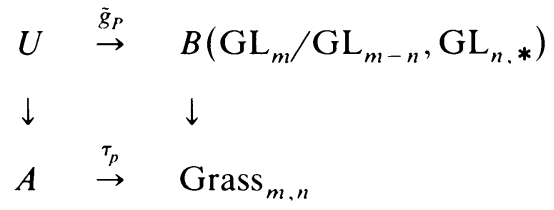

This follows from an explicit calculation with $\tilde{g}_{P}$ and $\tau_{P}$.

There is a similar result in the case of dimension 1. 
4.8 Proposition. If $X$ is a sscheme over $R$ of finite $\mathbf{Z} /$ l-cohomological dimension, there is a natural isomorphism

$$
\theta_{1}: \hat{K}_{1}^{\mathrm{et}}(X) \simeq \lim _{\rightarrow} \pi_{0} \operatorname{Hom}_{l}\left(X, \mathrm{GL}_{n}\right)_{R}
$$

$n$

Moreover, if $X=\operatorname{Spec} A$ is affine and $\alpha \in \mathrm{GL}_{n}(A)$ is an invertible $n \times n$ matrix over $A$, then $\theta_{1} \phi(\alpha)$ is represented by the map $\alpha^{\wedge}: A_{\mathrm{et}} \rightarrow\left(\mathrm{GL}_{n}\right)_{R}$.

Proof. Let $E \mathrm{GL}_{n}$ denote $B\left(\mathrm{GL}_{n}, \mathrm{GL}_{n, *}\right)[14,1.2]$, so that for each $n \geqslant 0$ there is a pullback diagram (of sschemes over $R$ ):

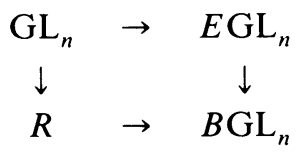

This becomes a homotopy fibre square of $l$-adic etale topological types and so gives a fibration sequence

$$
\operatorname{Hom}_{l}\left(X, \mathrm{GL}_{n}\right)_{R} \rightarrow \operatorname{Hom}_{l}\left(X, E \mathrm{GL}_{n}\right)_{R} \rightarrow \operatorname{Hom}_{l}\left(X, B \mathrm{GL}_{n}\right)_{R} \text {. }
$$

Since $E \mathrm{GL}_{n}$ is simplicially contractible to $R$, the space $\operatorname{Hom}_{l}\left(X, E \mathrm{GL}_{n}\right)_{R}$ is contractible and so the isomorphism $\theta_{1}$ can be constructed from Proposition 4.5 and the long exact homotopy sequence of a fibration.

In the case $X=\operatorname{Spec} A$, the natural maps of Proposition 2.5 determine a commutative diagram

$$
\begin{array}{ccccc}
\mathrm{GL}_{n}(A) & \rightarrow & \operatorname{Hom}\left(A, E \mathrm{GL}_{n}\right)_{R} & \rightarrow & B \mathrm{GL}_{n}(A) \\
\downarrow & & \downarrow & & \downarrow \\
\operatorname{Hom}_{l}\left(A, \mathrm{GL}_{n}\right)_{R} & \rightarrow & \operatorname{Hom}_{l}\left(A, E \mathrm{GL}_{n}\right)_{R} & \rightarrow & \operatorname{Hom}_{l}\left(A, B \mathrm{GL}_{n}\right)_{R}
\end{array}
$$

in which the rows are fibration sequences. By the argument of 4.5, the map $\phi$ : $K_{1}(A) \rightarrow \hat{K}_{1}^{\text {et }}(A)$ is equivalent to the abelianization of the direct limit of maps $\pi_{1}\left(B \mathrm{GL}_{n}(A)\right) \rightarrow \pi_{1} \operatorname{Hom}_{l}\left(A, B \mathrm{GL}_{n}\right)_{R}$ induced by the right vertical arrow above. It follows that $\theta_{1} \circ \phi$ is induced by the left vertical arrow above.

5. Relationship to cohomology. The purpose of this section is to construct a spectral sequence relating etale $K$-theory to etale cohomology. One of the major attractions of etale $K$-theory is the fact that in many cases it can be computed, either with the spectral sequence or by some other technique. Theorem 5.6 contains a periodicity statement for mod $l^{\nu}$ etale $K$-theory, which is proved by working with spectral sequence product structures.

As usual, $R$ denotes $\mathbf{Z}[1 / l]$. Since adjoining an $l$-primary root of unity to an $R$-algebra $A$ determines a finite etale extension, the sheaf $\mu_{l^{\nu}}$ of $l^{\nu}$ th roots of unity [28, II.2.18] is locally isomorphic to the constant sheaf $\mathbf{Z} / l^{\nu}$ for the etale topology on $R$. In particular, $\mu_{l}$ determines a coefficient system (see Definition 2.7) on $R_{\mathrm{et}}$, which for brevity is also denoted $\mu_{l^{\nu}}$. Let $\mathbf{Z} / l^{\nu}(0)$ denote the constant coefficient system $\mathbf{Z} / l^{\nu}$ and, for any $k>0$, let $\mathbf{Z} / l^{\nu}(k)$ be the coefficient system given by the $k$-fold tensor power of $\mu_{l}$. In addition, for any $k \geqslant 0$ let $\mathbf{Z}_{l}(k)$ be the pro-object of coefficient systems on $R_{\text {et }}$ given by $\left\{\mathbf{Z} / l^{\nu}(k)\right\}_{\nu>0}$. To simplify the statements that follow, we will use the convention that $\mathbf{Z} / l^{\nu}(k / 2)$ and $\mathbf{Z}_{l}(k / 2)$ are the zero coefficient systems unless $k$ is a nonnegative even integer. 
5.1 Proposition. Let $X$ be a connected sscheme over $R$ of finite $\mathbf{Z} /$ l-cohomological dimension. Then there is a natural, strongly convergent, fourth-quadrant spectral sequence

$$
E_{2}^{p,-q}=H_{\text {cont }}^{p}\left(X_{\mathrm{et}}, Z_{l}(q / 2)\right) \Rightarrow \hat{K}_{q-p}^{\mathrm{et}}(X) .
$$

Remark. Continuous cohomology was defined in Definition 2.8. Note that the natural summand of $\mathbf{Z}$ in $\hat{K}_{0}^{\mathrm{et}}(X)$ is $l$-adically completed in the abutment of this spectral sequence; in all other respects there is strong convergence in the ordinary sense.

Proof. Using Proposition 4.5 , interpret $\hat{K}_{*}^{\text {et }}(X)$ as $\pi_{*+n} \operatorname{Hom}_{l}\left(X, B \overline{\mathscr{G} \ell_{*}}\left(S^{n}\right)\right)_{R}$ for some $n>0$. It is clear from the proof of 4.5 that this involves the abovementioned $l$-adic completion when $*=0$. Consider the spectral sequence of Proposition 2.10

$$
\begin{aligned}
{ }^{\prime} E_{2}^{p,-q} & =H_{\mathrm{cont}}^{p}\left(X_{\mathrm{et}}, \Pi_{q} B \overline{\mathscr{G} \ell_{*}}\left(S^{n}\right)_{R}^{\wedge} / R_{\mathrm{et}}\right) \\
& \Rightarrow \pi_{q-p} \operatorname{Hom}_{t}\left(X_{\mathrm{et}}, B \overline{\mathscr{G} \ell_{*}}\left(S^{n}\right)_{R}^{\wedge}\right)_{R},
\end{aligned}
$$

where the basepoint is provided by the natural map $X \rightarrow R \rightarrow B \overline{\mathscr{G} \ell_{*}}\left(S^{n}\right)$. Define the spectral sequence $E_{r}^{*, *}$ by $E_{r}^{p,-q}={ }^{\prime} E_{r}^{p,-q-n}$. To identify the $E_{2}$-term, observe that the fibre of $\overline{B \mathscr{G} \ell_{*}}\left(S^{n}\right)_{R}^{\wedge} \rightarrow R_{\text {et }}$ is equivalent to the $n$-fold connective delooping of $\mathbf{Z}_{l}(0) \times B \mathrm{GL}_{\infty}(\mathbf{C})^{\wedge}$, so that the homotopy pro-groups of this fibre are abstractly isomorphic to $\left\{\mathbf{Z} / l^{\nu}\right\}_{\nu>0}$ in degrees of the form $n+2 k, k \geqslant 0$, and zero otherwise. The action of $\pi_{1} R_{\text {et }}$ on the pro-group in dimension $n+2 k$ is induced via delooping and the Hurewicz homomorphism by the action of $\pi_{1} R_{\mathrm{et}}$ on the $2 k$-dimensional fibre cohomology in the fibration

$$
B \mathrm{GL}_{k}(\mathbf{C})^{\wedge} \rightarrow\left(B \mathrm{GL}_{k}\right)_{R}^{\wedge} \rightarrow R_{\mathrm{et}} .
$$

Modulo decomposable elements, this fibre cohomology is generated by the $k$ th Chern class $c_{k}$; it follows from the fact that this class is algebraic that the dual homotopy pro-group affords the $\pi_{1} R_{\text {et }}$-representation $\mathbf{Z}_{l}(k)$. See [12, 5.5] for more details. The fact that $X$ has finite $\mathbf{Z} / l$-cohomological dimension implies that ${ }^{\prime} E_{2}{ }^{p}, q=0$ for $p$ sufficiently large, and so the convergence criterion of Proposition 2.10 is satisfied.

There is a similar spectral sequence for $\bmod l^{\nu}$ etale $K$-theory.

5.2 Proposition. Let $X$ be a connected sscheme over $R$ of finite $\mathbf{Z} /$ l-cohomological dimension. Then there is a natural, strongly convergent, fourth-quadrant spectral sequence

$$
E_{2}^{p,-q}=H^{p}\left(X_{\mathrm{et}}, \mathbf{Z} / l^{\nu}(q / 2)\right) \Rightarrow K_{q-p}^{\mathrm{et}}\left(X, \mathbf{Z} / l^{\nu}\right) .
$$

Proof. According to Definition $4.1, \S 3$, Proposition 4.5 the spectrum $W=\hat{\mathbf{K}}_{X}^{\text {et }}$ can be constructed from the collection of spaces $W_{i}, i \geqslant 0$, given by

$$
W_{i}=\operatorname{Hom}_{l}\left(X, B \overline{\mathscr{G} \ell} *\left(S^{i}\right)\right)_{R} .
$$

For each $n \geqslant 0$, let $W\langle n\rangle$ be the spectrum constructed from the collection of spaces $W\langle n\rangle_{i}, i \geqslant 0$, with

$$
W\langle n\rangle_{i}=\operatorname{Hom}_{t}\left(X_{\mathrm{et}},\left(B \overline{\mathscr{G} \ell_{*}}\left(S^{i}\right)_{R}^{\wedge}\right) / R_{\mathrm{et}}\langle n+i\rangle\right)_{R} .
$$


It follows from the various definitions that the spectral sequence of 5.1 for $\pi_{*} \hat{\mathbf{K}}_{X}^{\mathrm{ct}}$ is exactly the homotopy spectral sequence associated to the tower of spectra $\{W\langle n\rangle\}_{n \geqslant 0}$ by the procedure of $[3, \mathrm{IX}, 4.2]$. In particular, the $E_{2}$-formula in 5.1 is equivalent to a calculation of $\pi_{*}$ fibre $(W\langle n\rangle \rightarrow W\langle n-1\rangle), n \geqslant 0$, in terms of $H_{\text {cont }}^{*}\left(X_{\mathrm{et}}, \mathbf{Z}_{l}(n / 2)\right)$. The spectral sequence of this proposition is defined to be the homotopy spectral sequence of the tower $\{W\langle n\rangle \wedge \mathscr{M}(\nu)\}_{n \geqslant 0}$; it is straightforward to calculate the $E_{2}$-term by showing that for each $n \geqslant 0$ the homotopy groups of the fibre of the natural map $W\langle n\rangle \wedge \mathscr{M}(\nu) \rightarrow W\langle n-1\rangle \wedge \mathscr{M}(\nu)$ are given in terms of $H^{*}\left(X_{\mathrm{et}}, \mathbf{Z} / l^{\nu}(n / 2)\right)$.

The spectral sequences referred to in the following lemma are the spectral sequences of Proposition 2.10 in the special case in which all of the pro-spaces involved are trivial, i.e., are actual spaces.

5.3 Lemma. Let $g: V \rightarrow B$ and $h: W \rightarrow B$ be Kan fibrations with given sections and let $g \wedge h: V \wedge_{B} W \rightarrow B$ denote the mapping fibration of the fibrewise smash product of $g$ and $h$. For any $f: U \rightarrow B$, consider the associated smash product pairing of function spaces

$$
\operatorname{Hom}(U, V)_{B} \wedge \operatorname{Hom}(U, W)_{B} \rightarrow \operatorname{Hom}\left(U, V \wedge_{B} W\right)_{B},
$$

where the function spaces are pointed by the given sections. Then this pairing induces a pairing of homotopy spectral sequence

$$
\mu: E_{r}^{p,-q}(U, V) \otimes E_{r}^{p^{\prime},-q^{\prime}}(U, W) \rightarrow E_{r}^{p+p^{\prime},-q-q^{\prime}}\left(U, V \wedge_{B} W\right)
$$

with the following properties:

(1) On $E_{2}$, this pairing is induced by cup product in cohomology and smash product in homotopy of the fibres

$$
H^{p}\left(U, \Pi_{q} V / B\right) \otimes H^{p^{\prime}}\left(U, \Pi_{q^{\prime}} W / B\right) \rightarrow H^{p+p^{\prime}}\left(U, \Pi_{q+q^{\prime}} V \wedge_{B} W / B\right) .
$$

(2) For $v \in E_{r}^{p,-q}$ and $w \in E_{r}^{p^{\prime},-q^{\prime}}$,

$$
d_{r}(\mu(v \otimes w))=\mu\left(d_{r}(v) \otimes w\right)+(-1)^{p} \mu\left(v \otimes d_{r}(w)\right) .
$$

(3) The pairing on $E_{r+1}$ for $r \geqslant 2$ is induced by that on $E_{r}$, and the pairing on $E_{\infty}$ is induced by that on $E_{r}$ for all $r \geqslant 2$.

(4) The pairing on $E_{\infty}$ is compatible in the usual sense with the smash product pairing

$$
\pi_{q-p} \operatorname{Hom}(U, V)_{B} \otimes \pi_{q^{\prime}-p^{\prime}} \operatorname{Hom}(U, W)_{B} \rightarrow \pi_{q+q^{\prime}-p-p^{\prime}}\left(U, V \wedge_{B} W\right)_{B} .
$$

Proof. We will sketch the construction of a pairing satisfying (3) and (4), and omit the verification of (1) and (2) (see, for instance [4]).

For any fibration $Z \rightarrow B$ with given section and any pair $i, j$ with $0 \leqslant i \leqslant j$, let $Z\langle i, j\rangle$ denote the relative $(i-1)$-connected cover of the $j$ th Moore-Postnikov stage of $Z \rightarrow B$. More precisely, $Z\langle i, j\rangle$ lies in the pullback square (see 2.2)

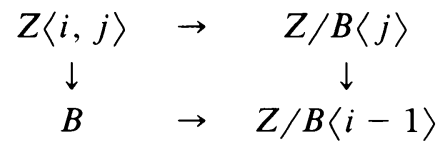


in which the lower horizontal map is induced by the given section $B \rightarrow Z$. By definition, the homotopy spectral sequence for $\operatorname{Hom}(U, Z)_{B}$ is the spectral sequence associated to the exact couple:

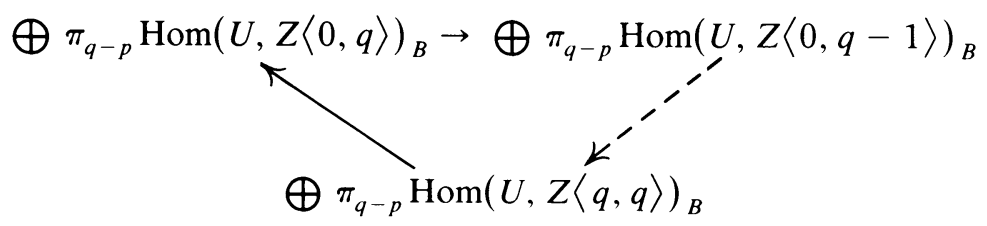

The $E_{r}^{p,-q}$-group in this spectral sequence is isomorphic to

$$
\text { image }\left\{\pi_{q-p} \operatorname{Hom}(U, Z\langle q, q+r-2\rangle)_{B} \rightarrow \pi_{q-p} \operatorname{Hom}(U, Z\langle q-r+2, q\rangle)_{B}\right\} \text {. }
$$

The pairing asserted in the lemma can therefore be derived in a straightforward way from the natural maps

$$
V\langle q, q+r-2\rangle \wedge_{B} W\left\langle q^{\prime}, q^{\prime}+r-2\right\rangle \rightarrow\left(V \wedge_{B} W\right)\left\langle q+q^{\prime}, q+q^{\prime}+r-2\right\rangle .
$$

5.4 Proposition. Let $X$ be a connected sscheme over $R$ of finite $\mathbf{Z} /$ l-cohomological dimension. Then for $l^{\nu} \neq 2$ there are natural bilinear pairings on the spectral sequences of Propositions 5.1 and 5.2. These pairings have the following properties:

(1) On $E_{2}$, the pairings are induced by cup product

$$
\begin{aligned}
& H_{\mathrm{cont}}^{p}\left(X_{\mathrm{et}}, \mathbf{Z}_{l}(q / 2)\right) \otimes H_{\mathrm{cont}}^{p^{\prime}}\left(X_{\mathrm{et}}, \mathbf{Z}_{l}\left(q^{\prime} / 2\right)\right) \rightarrow H_{\mathrm{cont}}^{p+p^{\prime}}\left(X_{\mathrm{et}}, \mathbf{Z}_{l}\left(q / 2+q^{\prime} / 2\right)\right), \\
& H^{p}\left(X_{\mathrm{et}}, \mathbf{Z} / l^{\nu}(q / 2)\right) \otimes H^{p^{\prime}}\left(X_{\mathrm{et}}, \mathbf{Z} / l^{\nu}\left(q^{\prime} / 2\right)\right) \rightarrow H^{p+p^{\prime}}\left(X_{\mathrm{et}}, \mathbf{Z} / l^{\nu}\left(q / 2+q^{\prime} / 2\right)\right) . \\
& \text { (2) and (3) As in (5.3). } \\
& \text { (4) The pairings on } E_{\infty} \text { are compatible with the pairings of } \S 4,
\end{aligned}
$$

$$
\begin{aligned}
\hat{K}_{i}^{\mathrm{et}}(X) \otimes \hat{K}_{j}^{\mathrm{et}}(X) & \rightarrow \hat{K}_{i+j}^{\mathrm{et}}(X), \\
K_{i}^{\mathrm{et}}\left(X, \mathbf{Z} / l^{\nu}\right) \otimes K_{j}^{\mathrm{et}}\left(X, \mathbf{Z} / l^{\nu}\right) & \rightarrow K_{i+j}^{\mathrm{et}}\left(X, \mathbf{Z} / l^{\nu}\right) .
\end{aligned}
$$

PROOF. The ring structures of $\S 4$ are derived from the object $\tilde{B} \overline{\mathscr{G} \ell} *$ of 3.6. There are maps

$$
B \overline{\mathscr{G} \ell_{*}}\left(S^{n}\right)^{\times 2} \leftleftarrows \tilde{B} \overline{\mathscr{G} \ell_{*}}\left(S^{n} \times S^{n}\right) \rightarrow B \overline{\mathscr{G} \ell_{*}}\left(S^{2 n}\right)
$$

of sschemes over $R$ which determine (up to homotopy) pairings

$$
B \overline{\mathscr{G} \ell_{*}}\left(S^{n}\right)_{R}^{\wedge} \wedge_{R_{\mathrm{et}}} B \overline{\mathscr{G} \ell_{*}}\left(S^{n}\right)_{R}^{\wedge} \rightarrow B \overline{\mathscr{G} \ell_{*}}\left(S^{2 n}\right)_{R}^{\wedge}
$$

of pro-spaces over $R_{\mathrm{et}}$. These pairings pass to maps $\operatorname{Hom}_{l}\left(X, B \overline{\mathscr{G} \ell_{*}}\left(S^{n}\right)\right)_{R} \wedge \operatorname{Hom}_{l}\left(X, B \overline{\mathscr{G} \ell_{*}}\left(S^{n}\right)\right)_{R} \rightarrow \operatorname{Hom}_{l}\left(X, B \overline{\mathscr{G} \ell_{*}}\left(S^{2 n}\right)\right)_{R}$ which (see 4.5 and [25, Appendix]) determine the ring structure of $\hat{K}_{*}^{\text {et }}(X)$. Consequently, the natural extension of 5.3 to pro-spaces over $R_{\mathrm{et}}$ gives a product structure on the spectral sequence of 5.1. The corresponding product structure on the spectral sequence of 5.2 can be obtained formally by working in the stable category (proof of 5.2) and using a suitable product map $\mathscr{M}(\nu) \wedge \mathscr{M}(\nu) \rightarrow \mathscr{M}(\nu)[1]$. 
Let $\zeta$ be a primitive $l^{\nu}$ th root of unity (if $l=2$ assume $\nu \geqslant 2$ ) and let $\beta_{\nu} \in$ $K_{2}\left(R(\zeta), \mathbf{Z} / l^{\nu}\right)$ be the image of $1 \bmod l^{\nu}$ under the map

$$
\mathbf{Z} / l^{\nu} \simeq \pi_{2}\left(B \mathbf{Z} / l^{\nu}, \mathbf{Z} / l^{\nu}\right) \rightarrow K_{2}\left(R(\zeta), \mathbf{Z} / l^{\nu}\right)
$$

induced by the homomorphism $\mathbf{Z} / l^{\nu} \rightarrow R(\zeta)^{*}=\mathrm{GL}_{1}(R(\zeta))$ sending 1 to $\zeta$.

REMARK. O. Gabber has pointed out that the proof of 8.4 below proceeds from an implicit assumption that the above map $\mathbf{Z} / l^{\nu} \rightarrow K_{2}\left(R(\zeta), \mathbf{Z} / l^{\nu}\right)$ is a group homomorphism. This can be verified if $l^{\nu} \neq 2$ by a straightforward calculation which compares homotopy to homology by the Hurewicz map.

We recall that, since $l^{\nu} \neq 2, R[\zeta]$ has finite $\mathbf{Z} / l$-cohomological dimension [37, III 1.3].

5.5 Lemma. The image of $\beta_{\nu}$ in $K_{2}^{\mathrm{et}}\left(R(\zeta), \mathbf{Z} / l^{\nu}\right)$ maps under the edge homomorphism of the spectral sequence of Proposition 5.2 to a generator of the cyclic group $H^{0}\left(R(\zeta)_{\mathrm{et}}, \mathbf{Z} / l^{\nu}(1)\right)$.

Proof. Let $A=R(\zeta)$. By naturality there is a commutative diagram

$$
\begin{array}{ccccc}
K_{2}\left(A, \mathbf{Z} / l^{\nu}\right) & \rightarrow & K_{1}(A) & \stackrel{l^{\nu}}{\rightarrow} & K_{1}(A) \\
\downarrow \phi & & \downarrow \phi & & \downarrow \phi \\
K_{2}^{\mathrm{et}}\left(A, \mathbf{Z} / l^{\nu}\right) & \rightarrow & \hat{K}_{1}^{\mathrm{et}}(A) & \stackrel{l^{\nu}}{\rightarrow} & \hat{K}_{1}^{\mathrm{et}}(A) \\
\downarrow & & \downarrow & & \downarrow \\
0 \rightarrow H^{0}\left(A_{\mathrm{et}}, \mathbf{Z} / l^{\nu}(1)\right) & \rightarrow & H_{\text {cont }}^{1}\left(A_{\mathrm{et}}, \mathbf{Z}_{l}(1)\right) \stackrel{l^{\nu}}{\rightarrow} H_{\text {cont }}^{1}\left(A_{\mathrm{et}}, \mathbf{Z}_{l}(1)\right)
\end{array}
$$

in which the lower vertical arrows are spectral sequence edge homomorphisms and the left-hand horizontal arrows are Bockstein homomorphisms. The rows in this diagram are exact (the lower one because $H_{\text {cont }}^{0}\left(A_{\mathrm{et}}, \mathbf{Z}_{l}(1)\right)$ vanishes [37, III.1.6]). It is easy to see that the maps in the center column are part of a commutative diagram:

$$
\begin{array}{ccc}
K_{1}(A) & \stackrel{\text { det }}{\rightarrow} & \operatorname{Hom}_{g}\left(A, \mathrm{GL}_{1}\right)_{R} \approx A^{*} \\
\downarrow \phi & \approx & \downarrow \\
K_{1}^{\mathrm{et}}(A) & \stackrel{\text { det }}{\rightarrow} & \pi_{0} \operatorname{Hom}_{l}\left(A, \mathrm{GL}_{1}\right)_{R} \approx H_{\text {cont }}^{1}\left(A_{\mathrm{et}}, Z_{l}(1)\right)
\end{array}
$$

Comparing the fibration sequences

$$
\begin{array}{ccccc}
\operatorname{Hom}_{g}\left(A, \mu_{l^{v}}\right)_{R} & \rightarrow & \operatorname{Hom}_{g}\left(A, \mathrm{GL}_{1}\right)_{R} & \stackrel{l^{\nu}}{\rightarrow} & \operatorname{Hom}_{g}\left(A, \mathrm{GL}_{1}\right)_{R} \\
\simeq \downarrow & & \downarrow & & \downarrow \\
\operatorname{Hom}_{l}\left(A, \mu_{l^{\nu}}\right)_{R} & \rightarrow & \operatorname{Hom}_{l}\left(A, \mathrm{GL}_{1}\right)_{R} & \stackrel{l^{\nu}}{\rightarrow} & \operatorname{Hom}_{l}\left(A, \mathrm{GL}_{1}\right)_{R}
\end{array}
$$

now leads directly to the conclusion that the $l^{\nu}$-torsion subgroup of $K_{1}(A)$ maps isomorphically above to the $l^{\nu}$-torsion subgroup of $H_{\text {cont }}^{1}\left(A_{\mathrm{et}}, \mathbf{Z}_{l}(1)\right)$. It follows that if $x$ is an element of $K_{2}\left(A, \mathbf{Z} / l^{\nu}\right)$ then the image of $x$ in $H^{0}\left(A_{\mathrm{et}}, \mathbf{Z} / l^{\nu}(1)\right)$ is a generator of this group if and only if the image of $x$ under the Bockstein homomorphism is a generator of the $l^{\nu}$-torsion subgroup of $K_{1}(A)$. By construction, $\beta_{\nu}$ has this second property. 
5.6 THEOREM. Let $\zeta$ be a primitive $l^{\nu}$ th root of unity, and let $X$ be a connected sscheme over $R(\zeta)$ of finite $\mathbf{Z} /$-cohomological dimension. If $l=2$, assume $\nu \geqslant 2$. Then the cup product map $\beta_{\nu}(-): K_{i}^{\mathrm{et}}\left(X, \mathbf{Z} / l^{\nu}\right) \rightarrow K_{i+2}^{\mathrm{et}}\left(X, \mathbf{Z} / l^{\nu}\right)$ is an isomorphism for $i \geqslant 0$.

5.7 REMARK. It is not hard to prove stronger periodicity results by exploiting the construction of "Bott elements" in [8]. For example, suppose that $l$ is odd, let $K$ be an algebraic number field and $A$ the integral closure of $R$ in $K$. Then if $X$ is a sscheme over $A$ of finite $\mathbf{Z} / l$-cohomological dimension, the groups $K_{i}^{\text {et }}\left(X, \mathbf{Z} / l^{\nu}\right)$ are periodic of period $2 d$, where $d$ is the degree of the field extension $K\left(\mu_{l^{\nu}}\right) / K$.

Proof of 5.6. Let $A=R(\zeta)$, and let $b_{\nu}$ be the image (see Lemma 5.5) of $\beta_{\nu}$ in $H^{0}\left(A_{\mathrm{et}}, \mathbf{Z} / l^{\nu}(1)\right)$. Since $b_{\nu}$ is a generator of this group (see 5.5), cup product with $b_{\nu}$ gives isomorphisms

$$
b_{\nu} \cup-: H^{i}\left(X_{\mathrm{et}}, \mathbf{Z} / l^{\nu}(k)\right) \approx H^{i}\left(X_{\mathrm{et}}, \mathbf{Z} / l^{\nu}(k+1)\right), \quad i, k \geqslant 0
$$

(see [37]). By 5.3, multiplication by $\beta_{\nu}$ on $K_{*}^{\text {et }}\left(X, \mathbf{Z} / l^{\nu}\right)$ corresponds on the $E_{2}$-level of the spectral sequence of 5.2 to the isomorphism given by multiplication by $b_{\nu}$. Inspection of the spectral sequence shows that multiplication by $\beta_{\nu}$ induces an isomorphism on the associated graded of $K_{*}^{\text {et }}\left(X, \mathbf{Z} / l^{\nu}\right)$ and therefore by $\beta_{\nu}$ on $K_{*}^{\mathrm{et}}\left(X, \mathbf{Z} / l^{\nu}\right)$ itself.

6. The transfer. This section contains a construction of the transfer map in algebraic $K$-theory and a parallel construction of the transfer map in etale $K$-theory. These transfer maps are induced by naturally defined maps of $\mathscr{F}$-spaces, and they commute with the natural transformation $\phi$ from algebraic $K$-theory to etale $K$-theory.

Throughout this section, $m$ will denote a fixed positive integer and $p: Y \rightarrow X$ an $m$-fold locally trivial covering of sschemes over $R$. (This means that $p$ is finite and etale of degree $m$ in each dimension and that the restriction of $p$ to some hypercovering $U \rightarrow X$ determines a degree $m$ covering map $\pi \cdot\left(U \times_{X} Y\right) \rightarrow \pi \cdot U$.)

Let $S$ denote the set $\{1, \ldots, m\}$ and $\Sigma=\Sigma_{m}$ the group of permutations of $S$. Let $\tilde{\Sigma}$ be the category with object set $\Sigma$ and exactly one morphism between any two objects; the nerve of $\tilde{\Sigma}$ is a contractible space $E \Sigma$ on which $\Sigma$ acts freely. For any subgroup $\rho \subseteq \Sigma$, space $A$ and space (respectively $R$-sscheme) $Z$ with left $\rho$-action, the symbol $A \times{ }_{\rho} Z$ (resp. $A \otimes_{\rho} Z$ ) will stand for the quotient of $A \times Z$ (resp. $A \otimes Z$ ) by the diagonal action of $\rho$.

6.1 Definition. Let $\rho$ be a subgroup of $\Sigma$. A classifying map for $p: Y \rightarrow X$ (with respect to $\rho$ ) is a geometric map (see 2.6) $f: X \rightarrow E \Sigma \otimes_{\rho} R$ which can be represented by an actual map $U \rightarrow E \Sigma \otimes_{\rho} R$ that fits into a cartesian square:

$$
\begin{array}{ccc}
U \times{ }_{X} Y & \rightarrow & (E \Sigma \times S) \otimes_{\rho} R \\
\downarrow & & \downarrow \\
U & \rightarrow & E \Sigma \otimes_{\rho} R
\end{array}
$$

Such a classifying map is obtained by choosing a hypercovering $U \rightarrow X$ which trivializes $p$ in each dimension and defining $U \rightarrow E \Sigma \otimes_{\rho} R$ to be the map over $R$ induced by any simplicial classifying map $\pi \cdot U \rightarrow E \Sigma / \rho$ of the simplicial covering $\pi \cdot\left(U \times_{X} Y\right) \rightarrow \pi \cdot U$. 
6.2 Proposition. Let $f: X \rightarrow-\rightarrow E \Sigma \otimes_{\rho} R$ be a classifying map for $p$. Then for any sscheme $Z$ over $R$ there is a natural commutative square

$$
\begin{array}{ccc}
\operatorname{Hom}_{g}\left(X, E \Sigma \otimes_{\rho} Z^{\times m}\right)_{E \Sigma \otimes_{\rho} R} & \stackrel{\psi}{\rightarrow} & \operatorname{Hom}_{g}(Y, Z)_{R} \\
\downarrow & & \downarrow \\
\operatorname{Hom}_{l}\left(X, E \Sigma \otimes_{\rho} Z^{\times m}\right)_{E \Sigma \otimes_{\rho} R} & \stackrel{\psi^{\mathrm{et}}}{\rightarrow} & \operatorname{Hom}_{l}(Y, Z)_{R}
\end{array}
$$

in which the vertical maps are of type in Proposition 2.5 and the map $\psi$ is an isomorphism. Moreover, if the natural map

$$
\left(Z^{\times m}\right)_{\mathrm{et}} \rightarrow Z_{\mathrm{et}} \times_{R_{\mathrm{ct}}} \cdots \times_{R_{\mathrm{ct}}} Z_{\mathrm{et}}
$$

becomes an equivalence after fibrewise l-adic completion over $R_{\mathrm{et}}$, then $\psi^{\mathrm{et}}$ is an equivalence.

Proof. If $U \rightarrow E \Sigma \otimes_{\rho} R$ represents $f$ and $U^{\prime}$ is a hypercovering of $X$ which refines $U$, then a $t$-simplex $U^{\prime} \otimes \Delta[t] \stackrel{h}{\rightarrow} E \Sigma \otimes_{\rho} Z^{\times m}$ is sent by $\psi$ to the composite

$$
\left(U^{\prime} \times_{X} Y\right) \otimes \Delta[t] \stackrel{\tilde{h}}{\rightarrow}(E \Sigma \times S) \otimes_{\rho} Z^{\times m} \stackrel{\text { proj }}{\rightarrow} Z
$$

where $\tilde{h}$ is defined by the cartesian square

$$
\begin{array}{ccc}
\left(U^{\prime} \times{ }_{X} Y\right) \otimes \Delta[t] & \stackrel{\tilde{h}}{\rightarrow} & (E \Sigma \times S) \otimes_{\rho} Z^{\times m} \\
\downarrow & & \downarrow \\
U^{\prime} \otimes \Delta[t] & \stackrel{h}{\rightarrow} & E \Sigma \otimes_{\rho} Z^{\times m}
\end{array}
$$

and proj is induced by the $\Sigma$-map $(E \Sigma \times S) \otimes Z^{\times m} \rightarrow Z$ sending $\left(\sigma, i, z_{1}, \ldots, z_{m}\right)$ to $z_{i}$. It is easy to check that this construction gives a bijection.

$$
\operatorname{Hom}\left(U^{\prime}, E \Sigma \otimes_{\rho} Z^{\times m}\right)_{E \Sigma \otimes_{\rho} R} \rightarrow \operatorname{Hom}\left(U^{\prime} \times_{X} Y, Z\right)_{R} .
$$

The fact that $\psi$ is an isomorphism is deduced by passing to the direct limit in $U^{\prime}$ and using the fact that any hypercovering $V$ of $Y$ is dominated by one of the form $U^{\prime} \times{ }_{X} Y$.

In the same vein, a map

$$
U_{\mathrm{et}}^{\prime} \times \Delta[t] \stackrel{k}{\rightarrow}\left(E \Sigma \otimes_{\rho} Z^{\times m}\right)_{E \Sigma \otimes_{\rho} R}^{\wedge}
$$

is sent by $\psi^{\text {et }}$ to the composite

$$
\left(U^{\prime} \times{ }_{X} Y\right)_{\mathrm{et}} \times \Delta[t] \stackrel{\tilde{k}}{\rightarrow}\left((E \Sigma \times S) \otimes_{\rho} Z^{\times m}\right)_{R}^{\wedge} \stackrel{\left(\text { proj }_{\text {et }}\right.}{\rightarrow} Z_{R}^{\wedge},
$$

where $\tilde{k}$ is defined by the cartesian square

$$
\begin{array}{ccc}
\left(U^{\prime} \times{ }_{X} Y\right)_{\mathrm{et}} \times \Delta[t] & \stackrel{\tilde{k}}{\rightarrow} & \left((E \Sigma \times S) \otimes_{\rho} Z^{\times m}\right)_{(E \Sigma \times S) \otimes_{\rho} R}^{\wedge} \\
\downarrow & & \downarrow \\
U_{\mathrm{et}}^{\prime} \times \Delta[t] & \stackrel{k}{\rightarrow} & \left(E \Sigma \otimes_{\rho} Z^{\times m}\right)_{E \Sigma \otimes_{\rho} R}^{\wedge}
\end{array}
$$


and (proj) $)_{\mathrm{et}}$ is derived from the map proj above. There is a map

$$
\operatorname{Hom}_{t}\left(X_{\mathrm{et}}, E \Sigma \times_{\rho}\left(Z_{R}^{\wedge}\right)^{\times m}\right)_{E \Sigma \times_{\rho} R_{\mathrm{ct}}} \rightarrow \operatorname{Hom}_{l}(Y, Z)_{R}
$$

defined in the same way as $\psi$, and it is easy to see that this map is an isomorphism. Moreover, if $Z$ satisfies the stated hypothesis, then by the product properties of the fibrewise completion functor [3] the natural map

$$
\left(E \Sigma \times_{\rho} Z^{\times m}\right)_{E \Sigma \otimes_{\rho} R}^{\wedge} \rightarrow E \Sigma \times_{\rho}\left(Z_{R}^{\wedge}\right)^{\times^{m}}
$$

is an equivalence. It follows then that the composition $\psi^{\text {et }}$ of the above constructed maps is an equivalence.

6.3 Proposition. Let $f: X \rightarrow E \Sigma \otimes_{\phi} R$ be a classifying map for $p: Y \rightarrow X$, and let $B \bar{G} \ell_{*}$ be the $\mathscr{F}-s s c h e m e$ over $R$ of Proposition 3.5. There is a naturally constructed commutative square of $\mathscr{F}$-spaces.

$$
\begin{array}{ccc}
\operatorname{Hom}_{g}\left(X, E \Sigma \otimes_{\rho} B \overline{\mathscr{G l}}{ }_{*}\right)_{E \Sigma \otimes_{\rho} R} & \stackrel{\theta}{\rightarrow} & \operatorname{Hom}_{g}(X, B \overline{\mathscr{G l}})_{R} \\
\downarrow & & \downarrow \\
\operatorname{Hom}_{l}\left(X, E \Sigma \otimes_{\rho} B \overline{\mathscr{G} \ell_{*}} \times m\right)_{E \Sigma \otimes_{\rho} R} & \stackrel{\theta^{\mathrm{cl}}}{\rightarrow} & \operatorname{Hom}_{l}\left(X, B \overline{\mathscr{G} \ell_{*}}\right)_{R}
\end{array}
$$

in which the vertical maps are of the type found in Proposition 2.5.

Proof. According to [23, p. 81] there is a $\Sigma$-equivariant sum functor $\tilde{\Sigma} \otimes \overline{\mathscr{G} \ell}_{*}^{\times m} \rightarrow$ $\overline{\mathscr{G}}_{\ell_{*}}$ where $\Sigma$ acts diagonally on the left and trivially on the right. Passing to the diagonal of the nerves of these sscheme-categories as in 3.5 gives a $\Sigma$-equivariant map $E \Sigma \otimes B \overline{\mathscr{G} \ell_{*}} \times m \rightarrow B \overline{\mathscr{G} \ell_{*}}$ which induces a map $T: E \Sigma \otimes_{\rho} B \overline{\mathscr{G} \ell_{*}} \times{ }^{m} \rightarrow B \overline{\mathscr{G} \ell_{*}}$ of $\mathscr{F}$-sschemes. The maps $\theta$ and $\theta^{\text {et }}$ are induced by $T$.

Propositions 6.2 and 6.3 lead to the transfer map.

6.4 THEOREM. If $X$ and $Y$ are affine, the diagram of $\mathscr{F}$-spaces

$$
\begin{aligned}
\operatorname{Hom}_{g}(Y, B \overline{\mathscr{G l}})_{R} \stackrel{\stackrel{\psi}{\rightleftarrows}}{ } \operatorname{Hom}_{g}\left(X, E \Sigma \otimes_{\rho} B \overline{\mathscr{G l}}{ }_{*}^{\times m}\right)_{E \Sigma \otimes_{\rho} R} \\
\stackrel{\theta}{\rightarrow} \operatorname{Hom}_{g}\left(X, B \overline{\mathscr{G l}}{ }_{*}^{\times m}\right)_{R}
\end{aligned}
$$

determines up to homotopy a map of spectra $p_{!}: \mathbf{K}_{Y} \rightarrow \mathbf{K}_{X}$. The corresponding diagram of F्F-spaces

$$
\begin{aligned}
& \operatorname{Hom}_{l}\left(Y, B \overline{\mathscr{G} \ell_{*}}\right)_{R} \stackrel{\stackrel{\psi^{\mathrm{et}}}{\rightleftarrows}}{ } \operatorname{Hom}_{l}\left(X, E \Sigma \otimes_{\rho} B \overline{\mathscr{G l}}{ }_{*}^{\times m}\right)_{E \Sigma \otimes_{\rho} R} \\
& \stackrel{\theta^{\mathrm{et}}}{\rightarrow} \operatorname{Hom}_{l}\left(X, B \overline{\mathscr{G} \ell_{*}}\right)_{R}
\end{aligned}
$$

determines up to homotopy a map of spectra $p_{!}^{\mathrm{et}}: \hat{\mathbf{K}}_{Y}^{\mathrm{et}} \rightarrow \hat{\mathbf{K}}_{X}^{\mathrm{et}}$. The maps $p$ ! and $p_{!}^{\mathrm{et}}$ fit into a homotopy-commutative square:

$$
\begin{array}{ccc}
\mathbf{K}_{Y} & \stackrel{p:}{\rightarrow} & \mathbf{K}_{X} \\
\downarrow \phi & & \downarrow \phi \\
\hat{\mathbf{K}}_{Y}^{\mathrm{et}} & \underset{p_{!}^{\mathrm{el}}}{\rightarrow} & \hat{\mathbf{K}}_{X}^{\mathrm{et}}
\end{array}
$$


In addition, $p$, and $p_{!}^{\text {et }}$ do not depend up to homotopy on the choice of a classifying map for $p: Y \rightarrow X$, and the map on homotopy groups induced by $p$ ! is the usual transfer map $p !: K_{*} Y \rightarrow K_{*} X[32, p .111]$.

Proof. The existence of $p_{!}, p_{!}^{\text {et }}$ and the commutativity statement are immediate from 6.2 and 6.3. To verify that $p_{!}^{\text {et }}$, say, is independent of the choice of the classifying map for $p: Y \rightarrow X$, consider two classifying maps $f, g: X-\rightarrow E \Sigma \otimes_{\phi} R$, together with the corresponding transfers $p_{!}^{\text {et }}(f), p_{!}^{\text {et }}(g)$. Since $f$ and $g$ are related by a simplicial homotopy $h: X \otimes \Delta[1] \rightarrow E \Sigma \otimes_{\phi} R$, the maps $p_{!}^{\text {et }}(f)$ and $p_{!}^{\text {et }}(g)$ are both deformation retracts of $p_{!}^{\text {et }}(h): \hat{\mathbf{K}}_{Y \otimes \Delta[1]}^{\text {et }} \rightarrow \hat{\mathbf{K}}_{X \otimes \Delta[1]}^{\text {et }}$.

Suppose that $X=\operatorname{Spec} A, Y=\operatorname{Spec} B$, and that $A^{\prime}$ is a finite etale $A$-algebra such that $B \otimes_{A} A^{\prime}$ is a disjoint union of $m$ copies of $B$. In this case the maps $\psi$ and $\theta$ are determined (via descent - see appendix) by maps

$$
\mathrm{GL}_{n}\left(B \otimes_{A} A^{\prime}\right) \rightarrow \Sigma_{m} \ltimes \mathrm{GL}_{n}\left(A^{\prime}\right)^{\times m} \rightarrow \mathrm{GL}_{m n}\left(A^{\prime}\right)
$$

where $\ltimes$ denotes semidirect product. This is one of the usual constructions of the Quillen transfer map [32, p. 111].

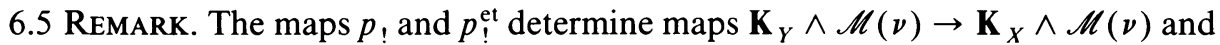
$\hat{\mathbf{K}}_{Y}^{\text {et }} \wedge \mathscr{M}(\nu) \rightarrow \hat{\mathbf{K}}_{X}^{\text {et }} \wedge \mathscr{M}(\nu)$. This gives transfer homomorphisms on etale or algebraic $K$-theory with coefficients $\bmod l^{\nu}$.

The following lemma is used in $§ 7$.

6.6 Lemma. If $p: Y \rightarrow X$ is a trivial covering (i.e., $Y$ is isomorphic via $p$ to a disjoint union of $m$ copies of $X)$, then the above transfer map $K_{*}^{\mathrm{et}}\left(Y, \mathbf{Z} / l^{\nu}\right) \rightarrow K_{*}^{\mathrm{et}}\left(X, \mathbf{Z} / l^{\nu}\right)$ is split surjective.

Proof. In this case $K_{*}^{\text {et }}\left(Y, \mathbf{Z} / l^{\nu}\right) \approx \oplus_{m} K_{*}^{\text {et }}\left(X, \mathbf{Z} / l^{\nu}\right)$, and it follows directly from the above constructions that the transfer map is the sum homomorphism

$$
\bigoplus_{m} K_{*}^{\mathrm{et}}\left(X, \mathbf{Z} / l^{\nu}\right) \stackrel{+}{\rightarrow} K_{*}^{\mathrm{et}}\left(X, \mathbf{Z} / l^{\nu}\right) .
$$

7. Galois descent and secondary transfer. Proposition 7.1 shows that etale $K$-theory satisfies descent for a general Galois cover. Theorem 7.4 constructs a secondary transfer map in algebraic $K$-theory or etale $K$-theory for a cyclic Galois cover-the indeterminacy of this secondary transfer map is the image of the (primary) transfer map constructed in $\S 6$. The section concludes with Theorem 7.9, which uses Galois descent over a tower of cyclic maps to show that the secondary transfer in etale $K$-theory is surjective for a cyclic map which belongs to such a tower.

Throughout this section, $m$ is a fixed positive integer and $p: Y \rightarrow X$ is a Galois map of sschemes over $R$ with Galois group $\Gamma \approx \mathbf{Z} / m$; in other words $p$ is finite, etale and principal with group $\Gamma$. If $\Gamma$ is treated as a subgroup of $\Sigma=\Sigma_{m}$ via the regular embedding, then the transfer machinery of $\S 6$ applies to this situation with $\rho=\Gamma$. If $Z$ is a sscheme over $R$, then for brevity $\overline{\mathbf{K}}_{Z}^{\text {et }}$ will denote the $\bmod l^{\nu}$ etale $K$-theory spectrum $\hat{\mathbf{K}}_{Z}^{\mathrm{et}} \wedge \mathscr{M}(\nu)$. 
7.1 Proposition. There are natural equivalences of spectra

$$
\hat{\mathbf{K}}_{X}^{\mathrm{et}} \underset{\Gamma}{\sim} \underset{\Gamma}{\operatorname{holim}} \hat{\mathbf{K}}_{Y}^{\mathrm{et}}, \quad \overline{\mathbf{K}}_{X}^{\mathrm{et}} \stackrel{\sim}{\rightarrow} \underset{\Gamma}{\operatorname{holim}} \overline{\mathbf{K}}_{Y}^{\mathrm{et}},
$$

where the action of $\Gamma$ on $\hat{\mathbf{K}}_{Y}^{\mathrm{et}}$ and on $\overline{\mathbf{K}}_{Y}^{\mathrm{et}}$ is that induced by the action of $\Gamma$ on $Y$.

Proof (cf. [8, Theorem 9]). Let $B$ denote the object $\left(B \overline{G \ell_{*}}\right)_{R}^{\wedge}$ over $R_{\mathrm{ct}}$. Since smashing with the finite complex $\mathscr{M}(\nu)$ commutes with taking stable homotopy inverse limiis, it is enough to verify the following chain of $\mathscr{F}$-space equivalences:

$$
\begin{aligned}
\operatorname{Hom}_{l}\left(X, B \overline{\mathscr{G} \ell_{*}}\right)_{R} & \equiv \operatorname{Hom}_{t}\left(X_{\mathrm{et}}, B\right)_{R_{\mathrm{ct}}} \\
& \sim \operatorname{Hom}_{t}\left(Y_{\mathrm{et}} \times{ }_{\Gamma} E \Gamma, B\right)_{R_{\mathrm{et}}} \\
& \sim \operatorname{Hom}^{\Gamma}\left(E \Gamma, \operatorname{Hom}_{l}\left(Y, B \overline{\mathscr{G} \ell_{*}}\right)_{R}\right) \\
& \sim \underset{\Gamma}{\operatorname{holim}} \operatorname{Hom}_{l}\left(Y, B \overline{\mathscr{G} \ell_{*}}\right)_{R} .
\end{aligned}
$$

The first row is the definition of $\operatorname{Hom}_{l}(\cdot, \cdot)$, the map of the second row is an equivalence induced by the equivalence $Y_{\mathrm{et}} \times_{\Gamma} E \Gamma \rightarrow X_{\mathrm{et}}$, the map in the third row comes from an adjointness construction and the definition of $\operatorname{Hom}_{l}(\cdot, \cdot)$, while the map in the bottom row is essentially the definition of holim $\Gamma$.

7.2 COROLlary. Let $X$ be a sscheme over $R$ of finite mod $l$ cohomological dimension. Suppose that $X \leftarrow X_{1} \leftarrow X_{2} \cdots \leftarrow X_{n} \leftarrow \cdots$ is an infinite sequence of maps such that each $X_{n}$ is Galois over $X$, each group $\Gamma_{n}=\operatorname{Gal}\left(X_{n} / X\right)$ is cyclic, and $\lim _{\rightarrow} v_{l}\left(\operatorname{order} \Gamma_{n}\right)=\infty$, where $v_{l}(\cdot)$ is the l-adic valuation. Then the natural map of spectra

$$
\overline{\mathbf{K}}_{X}^{\mathrm{et}} \rightarrow \lim _{\rightarrow} \overline{\mathbf{K}}_{X_{n}}^{\mathrm{et}}
$$

$n$

is equivalent to the inclusion of the homotopy fibre of the map

$$
1-\tilde{\gamma}: \underset{n}{\lim _{\rightarrow}} \overline{\mathbf{K}}_{X_{n}}^{\mathrm{et}} \rightarrow \underset{n}{\lim } \overline{\mathbf{K}}_{X_{n}}^{\mathrm{et}},
$$

where $\tilde{\gamma}$ is a topological generator of $\lim _{\leftarrow} \Gamma_{n}$.

Proof. The homotopy fibre of the map $1-\tilde{\gamma}$ is

$$
\underset{\mathbf{z}}{\operatorname{holim}}\left(\underset{n}{\lim } \overline{\mathbf{K}}_{X_{n}}^{\text {et }}\right)
$$

where the generator $g \in \mathbf{Z}$ acts on $\overline{\mathbf{K}}_{X_{n}}^{\text {et }}$ through $\mathbf{Z} \rightarrow \Gamma_{n}$ sending $g$ to the image of $\tilde{\gamma}$. By Proposition 7.1 it is enough to show that the natural map

$$
\underset{n}{\lim }\left(\underset{\Gamma_{n}}{\operatorname{holim}} \overline{\mathbf{K}}_{X_{n}}^{\mathrm{et}}\right) \rightarrow \underset{\mathbf{Z}}{\operatorname{holim}}\left(\underset{n}{\lim } \overline{\mathbf{K}}_{X_{n}}^{\mathrm{et}}\right)
$$


is a homotopy equivalence. For each $n$ there is a spectral sequence [3]

$$
E_{2}^{i,-j}(n)=H^{i}\left(\Gamma_{n}, K_{j}^{\mathrm{et}}\left(X_{n}, \mathbf{Z} / l^{\nu}\right)\right) \Rightarrow K_{j-i}^{\mathrm{et}}\left(X, \mathbf{Z} / l^{\nu}\right) .
$$

This spectral sequence converges strongly-in fact, it can be mapped to the Atiyah-Hirzebruch spectral sequence (see 5.2)

$$
E_{2}^{i,-j}=H^{i}\left(X_{\mathrm{et}}, \mathbf{Z} / l^{\nu}(j / 2)\right) \Rightarrow K_{j-i}^{\mathrm{et}}\left(X, \mathbf{Z} / l^{\nu}\right)
$$

in a way which covers the identity map on abutments. This implies that the $\mathbf{Z} / l$-cohomological dimension of $X$ gives a global bound (independent of $n$ ) on the filtration of elements in $E_{\infty}^{*, *}(n)$. It follows that there is a strongly convergent direct limit spectral sequence

$$
E_{2}^{i,-j}(\infty)=\lim _{\rightarrow} H^{i}\left(\Gamma_{n}, K_{j}^{\mathrm{et}}\left(X_{n}, \mathbf{Z} / l^{\nu}\right)\right) \Rightarrow K_{j-i}^{\mathrm{et}}\left(X, \mathbf{Z} / l^{\nu}\right) .
$$

The proof is completed by comparing this with the spectral sequence

$$
' E_{2}^{i,-j}(\infty)=H^{i}\left(\mathbf{Z}, \underset{n}{\lim } K_{j}^{\mathrm{et}}\left(X_{n}, \mathbf{Z} / l^{\nu}\right)\right) \Rightarrow \pi_{j-i} \underset{\mathbf{Z}}{\operatorname{holim}}\left(\underset{n}{\underset{n}{\lim }} \overline{\mathbf{K}}_{X_{n}}^{\mathrm{et}}\right)
$$

and using the fact that $\mathbf{Z} \rightarrow\left\{\Gamma_{n}\right\}$ is a $\mathbf{Z} /$ l-equivalence to conclude that $E_{2}^{i,-j}(\infty) \approx$ $' E_{2}^{i,-j}(\infty)$.

The construction of the secondary transfer depends upon a choice of homotopy. The following proposition makes that choice algebraically once and for all.

7.3 Proposition. A choice of generator $\gamma \in \Gamma$ determines a self-map of $\mathscr{F}$-sschemes.

$$
\tilde{\gamma}: E \Sigma \otimes_{\Gamma} B \overline{\mathscr{G l}}_{*}^{\times m} \rightarrow E \Sigma \otimes_{\Gamma} B \overline{\mathscr{G l}}_{*}^{\times m}
$$

with the property that

$$
\theta \circ \tilde{\gamma}=\gamma \circ \theta: \operatorname{Hom}_{g}\left(X, E \Sigma \otimes_{\Gamma} B \overline{\mathscr{G l}} \times_{*}\right)_{E \Sigma \otimes_{\Gamma} R} \rightarrow \operatorname{Hom}_{g}(Y, B \overline{\mathscr{G l}})_{R},
$$

where $\gamma: \operatorname{Hom}_{g}(Y, Z)_{R} \rightarrow \operatorname{Hom}_{g}(Y, Z)_{R}$ is induced by the action of $\gamma$ on $Y$. Similarly, $\theta^{\text {et }} \circ \tilde{\gamma}=\gamma \circ \theta^{\mathrm{et}}$. In addition, there is a natural simplicial homotopy

$$
H:\left(E \Sigma \otimes_{\Gamma} B \overline{\mathscr{G l}}_{*}^{\times m}\right) \otimes \Delta[1] \rightarrow E \Sigma \otimes_{\Gamma} B \overline{\mathscr{G l}}_{*}^{\times m}
$$

relating $\tilde{\gamma}$ to the identity map

Proof. Since $\Gamma$ is abelian, the action of $\gamma$ on $E \Sigma$ commutes with the action of $\Gamma$ on $E \Sigma$ and thus induces $\tilde{\gamma}$. The asserted equality $\theta \circ \tilde{\gamma}=\gamma \circ \theta$ is proved by observing that permuting the factors of $B \overline{\mathscr{G l}}_{*}^{\times m}$ and then projecting via $(E \Sigma \times S) \otimes_{\Gamma} B_{\mathscr{G} \ell_{*}}^{\times m}$ $\rightarrow B \overline{G \ell}$. has the effect of composing with an automorphism of $p: Y \rightarrow X$. There is a unique ( $\Gamma$-invariant) natural transformation from the identity functor $\tilde{\Sigma} \rightarrow \tilde{\Sigma}$ to the functor provided by multiplication by $\gamma$. This gives a $\Gamma$-invariant natural transformation

$$
\text { id } \rightarrow \gamma \otimes 1: \tilde{\Sigma} \otimes \overline{\mathscr{G}}_{*}^{\times m} \rightarrow \tilde{\Sigma} \otimes \overline{\mathscr{G}}_{*}^{\times m}
$$

which determines the homotopy $H$.

Recall (Theorem 6.4) that $p$ ! and $p_{!}^{\text {et }}$ stand for the geometric transfer maps $\mathbf{K}_{Y} \rightarrow \mathbf{K}_{X}$ and $\hat{\mathbf{K}}_{Y}^{\text {et }} \rightarrow \hat{\mathbf{K}}_{X}^{\text {et }}$ as well as for the corresponding homotopy group maps. 
7.4 TheOREM. If $p: Y \rightarrow X$ is a Galois map as above of affine schemes over $R$, then the simplicial homotopy of Proposition 7.3 determines a homotopy-commutative diagram of maps of spectra

$$
\begin{array}{ccc}
\mathbf{K}_{Y} & \stackrel{\tau}{\rightarrow} & \text { fibre }(p !) \\
\downarrow & & \downarrow \\
\hat{\mathbf{K}}_{Y}^{\text {et }} & \stackrel{\tau^{\text {et }}}{\rightarrow} & \text { fibre }\left(p_{!}^{\text {et }}\right)
\end{array}
$$

such that the composites

$$
\mathbf{K}_{Y} \stackrel{\tau}{\rightarrow} \operatorname{fibre}\left(p_{!}\right) \rightarrow \mathbf{K}_{Y}
$$

and

$$
\hat{\mathbf{K}}_{Y}^{\mathrm{et}} \stackrel{\tau^{\mathrm{et}}}{\rightarrow} \text { fibre } p ! \hat{\mathbf{K}}_{Y}^{\mathrm{et}}
$$

are both given by $1-\gamma$.

7.5 REMARK. The maps of 7.4 give rise to commutative diagrams:

$$
\begin{array}{ccc}
K_{n}(Y)^{\Gamma} & \stackrel{p_{! !}}{\rightarrow} & K_{n+1}(X) / \operatorname{image}\left(p_{!}\right) \\
\downarrow \phi & & \downarrow \phi \\
K_{n}^{\mathrm{et}}(Y)^{\Gamma} & \stackrel{p_{! 1}^{\mathrm{et}}}{\rightarrow} & K_{n+1}^{\mathrm{et}}(X) / \operatorname{image}\left(p_{!}\right)
\end{array}
$$

(Here $G^{\Gamma}$ denotes the subgroup of $\Gamma$-fixed elements in the group $G$.) The horizontal maps $p_{! !}$and $p_{! !}^{\text {et }}$ are called the secondary transfer homomorphisms associated to $p$.

7.6 REMARK. The above constructions are on the level of spectra, so that applying the functor $-\wedge \mathscr{M}(\nu)$ gives maps $\tau, \tau^{\text {et }}$ and secondary transfer homomorphisms with coefficients $\bmod l^{\nu}$.

Proof of TheOREM 7.4. There is a commutative diagram of $\mathscr{F}$-sschemes (see 7.3, proof of 6.3):

$$
\begin{aligned}
& E \Sigma \otimes_{\Gamma} B \overline{\mathscr{G} \ell}{ }_{*}^{\times m} \quad \underset{\dot{\gamma}}{\stackrel{1}{\rightrightarrows}} \quad E \Sigma \otimes_{\Gamma} B \overline{\mathscr{G} \ell}{ }_{*}^{\times m}
\end{aligned}
$$

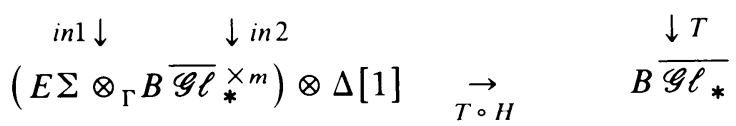

Applying $\operatorname{Hom}_{l}(X,-)_{(-)}$and taking the spectra associated to the resulting $\mathscr{F}$-spaces gives a commutative diagram of spectra

$$
\begin{aligned}
& \hat{\mathbf{J}}_{Y}^{\text {et }} \vee \hat{\mathbf{J}}_{Y}^{\text {et }} \stackrel{1+\bar{\gamma}}{\rightarrow} \quad \hat{\mathbf{J}}_{Y}^{\text {et }} \\
& \downarrow \text { in }^{\mathrm{et}} \quad \downarrow \theta^{\mathrm{et}} \\
& \hat{\mathbf{J}}_{Y}^{\mathrm{et}} \quad \underset{\theta^{\mathrm{et}} \circ H}{\rightarrow} \quad \hat{\mathbf{K}}_{X}^{\mathrm{et}}
\end{aligned}
$$


where $\psi^{\text {et. }}: \hat{\mathbf{J}}_{Y}^{\text {et }} \simeq \hat{\mathbf{K}}_{Y}^{\text {et }}$ and, similarly, ${ }^{\prime} \hat{\mathbf{J}}_{Y}^{\text {et }} \stackrel{\sim}{\rightarrow} \hat{\mathbf{K}}_{Y}^{\text {et }}$. The commutativity of this diagram determines a map fibre $\left(i{ }^{\text {et }}\right) \rightarrow$ fibre $\left(\theta^{\text {et }}\right)$. Define $\tau^{\text {et }}$ to be the composition

$$
\hat{\mathbf{K}}_{Y}^{\text {et }} \rightarrow \hat{\mathbf{J}}_{Y}^{\text {et }} \rightarrow \operatorname{fibre}\left(i^{\text {et }}\right) \rightarrow \operatorname{fibre}\left(\theta^{\text {et }}\right) \rightarrow \operatorname{fibre}\left(p_{!}^{\text {et }}\right),
$$

where the first map is the homotopy inverse of $\psi^{\text {et }}$, the second is the homotopy inverse of

$$
\text { fibre }\left(\text { in }^{\mathrm{et}}\right) \rightarrow \hat{\mathbf{J}}_{Y}^{\mathrm{et}} \vee \hat{\mathbf{J}}_{Y}^{\mathrm{et}} \stackrel{p r_{1}}{\rightarrow} \hat{\mathbf{J}}_{Y}^{\mathrm{et}}
$$

and the fourth comes from the definition of $p_{!}^{\text {et }}$ in terms of $\theta^{\text {et }}$.

The definition of $\tau$ is identical, with $\operatorname{Hom}_{l}(-,-)_{(-)}$replaced by $\operatorname{Hom}_{g}(-,-)_{(-)}$. The naturality condition of 2.5 produces the desired commutative diagrams.

The following is an analogue of Lemma 6.6.

7.7 Lemma. If $p: Y \rightarrow X$ is a trivial cyclic covering (i.e., $Y$ is isomorphic, via $p$, to a disjoint union of $m$ copies of $X$ ) then the map

$$
\tau^{\text {et }} \wedge \mathscr{M}(\nu): \overline{\mathbf{K}}_{Y}^{\mathrm{et}} \rightarrow \operatorname{fibre}\left(p_{!}^{\mathrm{et}}\right) \wedge \mathscr{M}(\nu)
$$

is surjective on homotopy.

Proof. As in 6.6 there is an isomorphism

$$
K_{*}^{\mathrm{et}}\left(Y, \mathbf{Z} / l^{\nu}\right) \approx \bigoplus_{m} K_{*}^{\mathrm{et}}\left(X, \mathbf{Z} / l^{\nu}\right)
$$

such that the action of $\Gamma$ on the direct sum is by cyclic permutation. The lemma is proved by inspecting the diagram

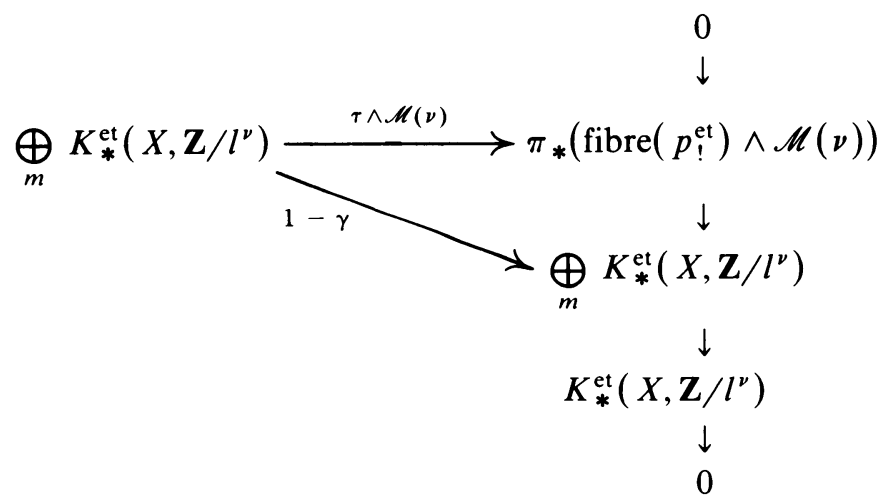

and using the fact that the right-hand column is exact.

One more fact is needed for the proof of 7.9. Suppose that

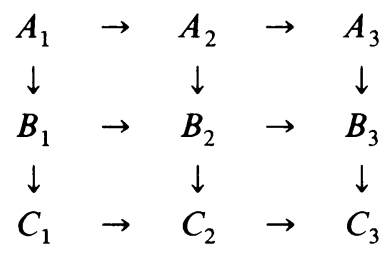


is a commutative diagram of spaces (or spectra) in which each row and each column is a fibration sequence. Let $\partial_{h}: \pi_{*} A_{3} \rightarrow \pi_{*-1} A_{1}$ be the connecting homomorphism for the topmost horizontal fibration sequence, and $\partial_{v}: \pi_{*} C_{1} \rightarrow \pi_{*-1} A_{1}$ the connecting homomorphism for the left-hand vertical fibration sequence. Let $F$ be the fibre of the evident map $B_{2} \rightarrow C_{3}$, and note that by assumption there are natural maps $f: F \rightarrow A_{3}, g: F \rightarrow C_{1}$.

7.8 Lemma. In the above situation, suppose that $x \in \pi_{i} F$. Then $\partial_{h} f(x)= \pm \partial_{r} g(x)$ in $\pi_{i-\mathrm{i}} A_{1}$.

Progf. If is not hard to see that up to homotopy there is a fibration sequence $A_{1} \rightarrow F \stackrel{(f, g)}{\rightarrow} A_{3} \times C_{1}$ for which the connecting homomorphism $\partial: \pi_{i} A_{3} \times \pi_{i} C_{1} \rightarrow$ $\pi_{i-1} A_{1}$ is given by the formula $\partial(u, w)=\partial_{h}(u) \pm \partial_{v}(w)$.

7.9 TheOREm. Let $X$ be a sscheme over $R$ of finite $\bmod l$ etale cohomological dimension. Suppose that

$$
X \stackrel{p}{\leftarrow} Y=X_{1} \leftarrow X_{2} \leftarrow \cdots \leftarrow X_{n} \leftarrow \cdots
$$

is an infinite sequence of maps such that each $X_{n}$ is Galois over $X$, each group $\Gamma_{n}=\operatorname{Gal}\left(X_{n} / X\right)$ is cyclic and $\lim _{n} v_{l}\left(\operatorname{order} \Gamma_{n}\right)=\infty$. Then the secondary transfer homomorphism (see 7.5, 7.6)

$$
p_{! !}^{\mathrm{et}}: K_{i-1}^{\mathrm{et}}\left(Y, \mathbf{Z} / l^{\nu}\right)^{\Gamma} \rightarrow K_{i}^{\mathrm{et}}\left(X, \mathbf{Z} / l^{\nu}\right) / \operatorname{image}\left(p_{!}^{\mathrm{et}}\right)
$$

is surjective for each $i \geqslant 1$.

Proof. Let $Y_{n}=X_{n} \times{ }_{X} Y$, so that there is an infinite tower of cartesian squares

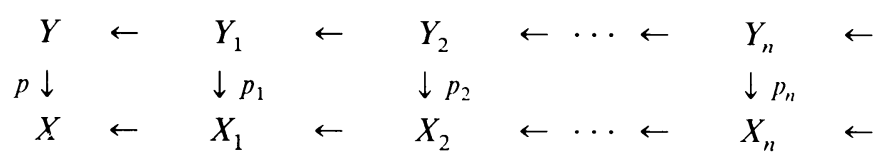

in which each $p_{n}: Y_{n} \rightarrow X_{n}(n \geqslant 1)$ is a trivial covering. By 7.2 and naturality, there is a $3 \times 3$ diagram of spectra

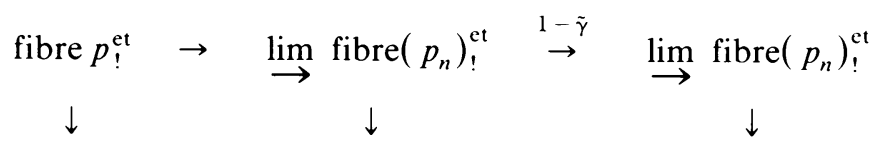

$$
\begin{aligned}
& \overline{\mathbf{K}}_{Y}^{\mathrm{et}} \quad \rightarrow \quad \lim _{\downarrow} \overline{\mathbf{K}}_{Y_{n}}^{\mathrm{et}} \quad \stackrel{1-\tilde{\gamma}}{\rightarrow} \quad \lim _{\boldsymbol{l}} \overline{\mathbf{K}}_{Y_{n}}^{\mathrm{et}} \\
& \downarrow p_{!}^{\mathrm{c} !} \quad \downarrow \lim _{\rightarrow}\left(p_{n}\right)_{!}^{\mathrm{et}} \quad \downarrow \lim _{\rightarrow}\left(p_{n}\right)_{!}^{\mathrm{et}} \\
& \overline{\mathbf{K}}_{X}^{\mathrm{et}} \quad \rightarrow \quad \lim _{\rightarrow} \overline{\mathbf{K}}_{X_{n}}^{\mathrm{et}} \quad \stackrel{1-\bar{\gamma}}{\rightarrow} \quad \stackrel{\lim }{\rightarrow} \overline{\mathbf{K}}_{X_{n}}^{\mathrm{et}}
\end{aligned}
$$

in which each row and each column is a fibration sequence, where $\tilde{\gamma}$ is a topological generator of $\lim _{\leftarrow} \Gamma_{n}$. For brevity we will use the notation of 7.8 to refer to the spectra in this diagram. Pick $x \in K_{i}^{\text {et }}\left(X, \mathbf{Z} / l^{\nu}\right)=\pi_{i} C_{1}$. By 6.6 the image of $x$ in $\pi_{i} C_{2}$ lifts to an elemeni of $\pi_{i} B_{2}$ and therefore (by an easy diagram chase) to an element $y$ of $\pi_{i} F$ such that $f(y)=x$. By 7.8, $\partial_{h} g(y)= \pm \partial_{l}(x)$. However, by 7.4 and a careful 
naturality argument, there is a map of fibration sequences:

$$
\begin{array}{lllll}
\overline{\mathbf{K}}_{Y}^{\mathrm{et}} & \rightarrow & \underset{\lim ^{\mathrm{lim}}}{\rightarrow} \overline{\mathbf{K}}_{Y_{n}}^{\mathrm{et}} & \stackrel{1-\tilde{\gamma}}{\rightarrow} & \stackrel{\lim }{\rightarrow} \overline{\mathbf{K}}_{Y_{n}}^{\text {et }} \\
\downarrow \tau^{\mathrm{et}} & & \downarrow \lim _{n}^{\mathrm{et}} & & \downarrow \underset{\rightarrow}{\rightarrow} \tau_{n}^{\text {et }} \\
A_{1} & \rightarrow & A_{2} & \rightarrow & A_{3}
\end{array}
$$

By 7.7 the element $g(y) \in \pi_{i} A_{3}$ can be lifted to an element $z \in \pi_{i} \lim \overline{\mathbf{K}}_{Y_{n}}^{\text {et }}$. The image of $z$ in $\pi_{i-1} \overline{\mathbf{K}}_{Y}^{\text {et }}=K_{i-1}^{\text {et }}\left(Y, \mathbf{Z} / l^{\nu}\right)$ then passes to $\pm x$ modulo image $\left(p_{!}^{\text {et }}\right)$ under the secondary transfer homomorphism.

8. Surjectivity theorems. In this section, we will prove the theorems announced in [7]. The basic result is the statement 8.5 that the natural map from algebraic $K$-theory $\bmod l^{\nu}$ to etale $K$-theory $\bmod l^{\nu}$ is surjective for fields of $\mathbf{Z} / l$-cohomological dimension $\leqslant 2$ and for rings of $S$-integers in global fields. For a finite field this map is an isomorphism (see 8.6). The corresponding $l$-adic surjectivity results $(8.7$, 8.9) are related to conjectures of S. Lichtenbaum. The section ends with a divisibility theorem (see 8.10). The reader familiar with [37] will recognize that the strategy below is just to combine results from the preceding sections with the arguments of C. Soulé.

Recall that a global field is either a number field (i.e., a finite algebraic extension of the rational number field $\mathbf{Q}$ ) or a function field (i.e., a finite algebraic uxtension of $\mathbf{F}_{p}(t)$ for some prime $p$ ). Let $\mathrm{cd}_{l}(-)$ denote (etale) $\mathbf{Z} / l$-cohomological dimension. If $F$ is a global field, it is known [36, II, 4.4] that $\mathrm{cd}_{l}(F) \leqslant 2$ under any of the following assumptions:

(i) $F$ is a number field and $l$ is odd,

(ii) $F$ is a number field with no real embeddings and $l=2$,

(iii) $F$ is a function field and $1 / l \in F$.

The surjectivity results below therefore apply to any global field which satisfies (i), (ii), or (iii), as well as to any other field $F$ with $\mathrm{cd}_{l}(F) \leqslant 2$, such as a finite field, a nonarchimedean completion of a number field, or the function field of a surface over a separably closed field of characteristic different from $l$.

A ring of $S$-integers $A$ in a global field $F$ is a finitely-generated Dedekind domain with field of fractions $F$. If $F$ is a number field let $X=\operatorname{Spec} \mathcal{O}$, where $\mathcal{O}$ is the integral closure of $\mathbf{Z}$ in $F$, while if $F$ is a function field let $X$ be its associated smooth complete curve. In either case $\operatorname{Spec} A$ is obtained from $X$ by deleting a finite set of closed points (a set which must be nonempty in the function field case). The localization sequence in etale cohomology [37, III.1] implies that a ring of $S$-integers $A$ in a global field $F$ satisfies $\operatorname{cd}(A) \leqslant 2$ if $\operatorname{cd}(F) \leqslant 2$.

Etale $K$-theory starts out with schemes over $R=\mathbf{Z}[1 / l]$. The following well-known lemma shows that from the algebraic $K$-theory point of view this is often no real restriction.

8.1 Proposition. Let $A$ be a ring of $S$-integers in a number field. Then the natural map

$$
K_{i}\left(A, \mathbf{Z} / l^{\nu}\right) \rightarrow K_{i}\left(A[1 / l], \mathbf{Z} / l^{\nu}\right)
$$

is an isomorphism for $i>1$ and an injection for $i=1$. 
Proof. Apply Quillen's localization sequence [32]

$$
\cdots \rightarrow K_{i}\left(A, \mathbf{Z} / l^{\nu}\right) \rightarrow K_{i}\left(A[1 / l], \mathbf{Z} / l^{\nu}\right) \rightarrow \bigoplus_{\omega \mid l} K_{i-1}\left(k_{\omega}, \mathbf{Z} / l^{\nu}\right) \rightarrow \cdots
$$

in which each $k_{\omega}$ is a finite field of characteristic $l$, so that $K_{i}\left(k_{\omega}, \mathbf{Z} / l^{\nu}\right)=0$ for $i>0[30]$.

What follows is a three-step proof of surjectivity, first in low dimensions, then in the presence of some roots of unity, and finally in general.

8.2 Proposition. Suppose that $A$ is either a field $F$ satisfying $1 / l \in F$ and $\mathrm{cd}_{l}(F) \leqslant 2$, or a ring of $S$-integers in a global field $F$ satisfying these conditions. Then the natural map (see 4.4)

$$
K_{i}\left(A[1 / l], \mathbf{Z} / l^{\nu}\right) \rightarrow K_{i}^{\mathrm{et}}\left(A[1 / l], \mathbf{Z} / l^{\nu}\right)
$$

is an isomorphism for $i=1$ or $i=2$.

8.3 REMARK. The above map is not necessarily an isomorphism for $i=0$. This fails even for $A=\mathbf{Q}$.

Proof of 8.2. Let $B$ denote $A[1 / l]$. By calculation (see $[29, \S \S 1,16]$ and the appendix to this paper) there are isomorphisms:

$$
\begin{aligned}
K_{0}^{\text {red }} B \equiv \operatorname{ker}\left(K_{0} B \stackrel{\text { rank }}{\rightarrow} \mathbf{Z}\right) & \stackrel{\text { det }}{\rightarrow} \text { Pic } B \approx \pi_{0} \operatorname{Hom}_{g}\left(B, B \mathrm{GL}_{1}\right)_{R}, \\
K_{1} B & \stackrel{\text { det }}{\rightarrow} \quad B^{*} \approx \pi_{1} \operatorname{Hom}_{g}\left(B, B \mathrm{GL}_{1}\right)_{R}
\end{aligned}
$$

Since cd ${ }_{l}(B) \leqslant 2$ and det: $\left(B \mathrm{GL}_{n}\right)_{\mathrm{et}} \rightarrow\left(B \mathrm{GL}_{1}\right)_{\mathrm{et}}$ is in low dimensions a fibrewise $l$-equivalence over $R_{\text {et }}$, there are isomorphisms (see 4.5):

$$
\begin{aligned}
\operatorname{ker}\left(\hat{K}_{0}^{\text {et }} B \stackrel{\text { rank }}{\rightarrow} \mathbf{Z}\right) & \stackrel{\text { det }}{\rightarrow} \pi_{0} \operatorname{Hom}_{l}\left(B, B \mathrm{GL}_{1}\right)_{R}, \\
\hat{K}_{1}^{\text {et }} B & \rightarrow \pi_{1} \operatorname{Hom}_{l}\left(B, B \mathrm{GL}_{1}\right)_{R}
\end{aligned}
$$

It follows that the map $K_{1}\left(B, \mathbf{Z} / l^{\nu}\right) \rightarrow K_{1}^{\text {et }}\left(B, \mathbf{Z} / l^{\nu}\right)$ is an isomorphism, since it can be identified with the connected component map induced by the left-hand vertical arrow in the following diagram (see 2.5) of fibrations:

$$
\begin{array}{ccccc}
\operatorname{Hom}_{g}\left(B, B \mu_{l^{\nu}}\right)_{R} & \rightarrow & \operatorname{Hom}_{g}\left(B, B \mathrm{GL}_{1}\right)_{R} & \stackrel{l^{\nu}}{\rightarrow} & \operatorname{Hom}_{g}\left(B, B \mathrm{GL}_{1}\right)_{R} \\
\simeq \downarrow & & \downarrow & & \downarrow \\
\operatorname{Hom}_{l}\left(B, B \mu_{l^{\nu}}\right)_{R} & \rightarrow & \operatorname{Hom}_{l}\left(B, B \mathrm{GL}_{1}\right)_{R} & \stackrel{l^{\nu}}{\rightarrow} & \operatorname{Hom}_{l}\left(B, B \mathrm{GL}_{1}\right)_{R}
\end{array}
$$

(The main step in this identification is to use the determinant function to produce a map from $\mathbf{K}_{B}$ to the spectrum determined by the tensor product multiplication on $Z \times \operatorname{Hom}_{g}\left(B, B \mathrm{GL}_{1}\right)_{R}$, as well as a map from $\hat{K}_{B}^{\text {et }}$ to the spectrum determined by the tensor product multiplication on $\mathbf{Z} \times \operatorname{Hom}_{l}\left(B, B \mathrm{GL}_{1}\right)_{R}$.)

The map of 4.4 together with the edge homomorphisms in the spectral sequence of 5.2 induces a map of short exact sequences:

$$
\begin{array}{ccccc}
0 \rightarrow K_{2}(B) \otimes \mathbf{Z} / l^{\nu} & \rightarrow & K_{2}\left(B, \mathbf{Z} / l^{\nu}\right) & \rightarrow & { }^{\nu} K_{1}(B) \rightarrow 0 \\
\downarrow f & & \downarrow g & & \\
0 \rightarrow H^{2}\left(B_{\mathrm{et}}, \mathbf{Z} / l^{\nu}(2)\right) & \rightarrow & K_{2}^{\mathrm{et}}\left(B, \mathbf{Z} / l^{\nu}\right) & \rightarrow & H^{0}\left(B_{\mathrm{et}}, \mathbf{Z} / l^{\nu}(1)\right) \rightarrow 0
\end{array}
$$


The map $h$ is the obvious isomorphism (see above), and the multiplicative properties of the spectral sequence (see 5.4) imply that the map $f$ is the Galois symbol [40,3.3]. The Galois symbol is known to be an isomorphism for any field [27]; this finishes the proof of Proposition 8.2 in the field case. If $A$ is a ring of $S$-integers, the proof is completed by applying verbatim the argument of [37, Lemma 10].

8.4 Proposition. Suppose that $A$ is either a field $F$ satisfying $1 / l \in F$ and $\operatorname{cd}_{l}(F) \leqslant 2$, or a ring of $S$-integers in a global field $F$ satisfying these conditions. Then if $A$ contains a primitive $l^{\nu}$ th root of unity and $l^{\nu} \neq 2$, the map

$$
K_{i}\left(A[1 / l], \mathbf{Z} / l^{\nu}\right) \rightarrow K_{i}^{\mathrm{et}}\left(A[1 / l], \mathbf{Z} / l^{\nu}\right)
$$

is naturally split surjective for $i \geqslant 1$. In particular, the splitting is preserved by the action of $\operatorname{Aut}(A)$ on the groups involved.

Proof. This follows immediately from 5.6, 8.2 and the multiplicative properties of the $\operatorname{map} K_{*}\left(A[1 / l], \mathbf{Z} / l^{\nu}\right) \rightarrow K_{*}^{\text {et }}\left(A[1 / l], \mathbf{Z} / l^{\nu}\right)$.

8.5 THEOREM. Suppose that $A$ is either a field $F$ satisfying $1 / l \in F$ and $\operatorname{cd}_{l}(F) \leqslant 2$, or a ring of $S$-integers in a global field $F$ satisfying these conditions. If $l=2$, assume that $\nu>1$ and that $A$ contains a primitive 4 th root of unity. Then the natural map

$$
K_{i}\left(A[1 / l], \mathbf{Z} / l^{\nu}\right) \rightarrow K_{i}^{\mathrm{et}}\left(A[1 / l], \mathbf{Z} / l^{\nu}\right)
$$

is surjective for $i \geqslant 1$.

Proof. Let $B$ denote $A[1 / l]$, and $B \rightarrow B^{\prime}$ the cyclic Galois extension obtained by adjoining a primitive $l^{\nu}$ th root of unity to $B$; note that successively adjoining primitive $l^{k \cdot v}$ th roots of unity to $B^{\prime}$ determines a tower as in 7.9. By 6.4 and 8.4, the image of $K_{i}\left(B, \mathbf{Z} / l^{\nu}\right) \rightarrow K_{i}^{\mathrm{et}}\left(B, \mathbf{Z} / l^{\nu}\right)$ contains the image of the transfer map $K_{i}^{\text {et }}\left(B^{\prime}, \mathbf{Z} / l^{\nu}\right) \rightarrow K_{i}^{\text {et }}\left(B, \mathbf{Z} / l^{\nu}\right)$. By 7.9 , the cokernel of this transfer map is in the image of the corresponding secondary transfer map. The theorem now follows from 7.4 and 8.4 .

8.6 COROLlaRY. If $\mathbf{F}_{q}$ is a finite field of characteristic different from $l$, then the natural maps

$$
K_{i}\left(\mathbf{F}_{q}, \mathbf{Z} / l^{\nu}\right) \rightarrow K_{i}^{\mathrm{et}}\left(\mathbf{F}_{q}, \mathbf{Z} / l^{\nu}\right), \quad K_{i}\left(\mathbf{F}_{q}\right) \otimes \mathbf{Z}_{l} \rightarrow \hat{K}_{i}^{\mathrm{et}}\left(\mathbf{F}_{q}\right)
$$

are isomorphisms for $i \geqslant 0$.

Proof. The case $i=0$ is trivial, so assume $i$ positive. Because $\operatorname{cd}_{l}\left(\mathbf{F}_{q}\right)=1$, the spectral sequence of 5.1 implies that $K_{2 j}^{\mathrm{et}}\left(\mathbf{F}_{q}, \mathbf{Z} / l^{\nu}\right) \approx H^{0}\left(\left(\mathbf{F}_{q}\right)_{\mathrm{et}}, \mathbf{Z} / l^{\nu}(j)\right)$ and $K_{2 j-1}^{\text {et }}\left(\mathbf{F}_{q}, \mathbf{Z} / l^{\nu}\right) \approx H^{1}\left(\left(\mathbf{F}_{q}\right)_{\mathrm{e}}, \mathbf{Z} / l^{\nu}(j)\right)$. These groups are isomorphic to the known finite groups $K_{2 j}\left(\mathbf{F}_{q}, \mathbf{Z} / l^{\nu}\right)$ and $K_{2 j-1}\left(\mathbf{F}_{q}, \mathbf{Z} / l^{\nu}\right)$ [30, 5]. This implies that the surjection $K_{i}\left(\mathbf{F}_{q}, \mathbf{Z} / l^{\nu}\right) \rightarrow K_{i}^{\text {et }}\left(\mathbf{F}_{q}, \mathbf{Z} / l^{\nu}\right)$ of 8.5 is an isomorphism. (There are some minor points here if $l=2$. First of all, if $l^{\nu} \geqslant 4$ the argument of 8.5 goes through verbatim for $\mathbf{F}_{q}$ even in the absence of a primitive fourth root of unity, since every degree 2 extension of a finite field is contained in a $\mathbf{Z}_{2}$-tower. Secondly, isomorphism in the case $l^{\nu}=2$ can be derived from isomorphism in the case $l^{\nu}=4$ by a universal coefficient exact sequence argument.) The proof is finished by using the 
fact that $K_{i}\left(\mathbf{F}_{q}\right)$ and $K_{i}^{\mathrm{et}}\left(\mathbf{F}_{q}\right)$ are finite groups to construct a chain of isomorphisms

$$
K_{i}\left(\mathbf{F}_{q}\right) \otimes \mathbf{Z}_{l} \approx \lim _{\leftarrow} K_{i}\left(\mathbf{F}_{q}, \mathbf{Z} / l^{\nu}\right) \approx \lim _{\leftarrow} K_{i}^{\mathrm{et}}\left(\mathbf{F}_{q}, \mathbf{Z} / l^{\nu}\right) \approx K_{i}^{\mathrm{et}}\left(\mathbf{F}_{q}\right) .
$$

8.7 Theorem. Suppose that $A$ is the ring of integers in a number field. If $l=2$, assume that $\sqrt{-1} \in A$. Then the natural map

$$
K_{n}(A) \otimes \mathbf{Z}_{l} \rightarrow \hat{K}_{n}^{\mathrm{et}}(A[1 / l])
$$

is a surjective for $n \geqslant 2$.

8.8 REMARK. The spectral sequence of 5.1 shows that there is an isomorphism

$$
\hat{K}_{n}^{\mathrm{et}}(A[1 / l]) \approx H_{\text {cont }}^{j}\left(A[1 / l]_{\mathrm{et}}, \mathbf{Z}_{l}(i)\right)
$$

where $n=2 i-j, j=1,2$. In view of the fact that the groups involved are finitelygenerated $\mathbf{Z}_{l}$-modules, this implies that the conjecture of $[33, \S 9]$ is equivalent to the conjecture that the surjection of 8.7 be an isomorphism.

Proof of 8.7. Construct a chain of maps

$$
\begin{aligned}
K_{n}(A) \otimes \mathbf{Z}_{l} & \rightarrow \lim K_{n}\left(A, \mathbf{Z} / l^{\nu}\right) \rightarrow \lim K_{n}\left(A[1 / l], \mathbf{Z} / l^{\nu}\right) \\
& \rightarrow \underset{\lim }{\leftarrow} K_{n}^{\mathrm{et}}\left(A[1 / l], \mathbf{Z} / l^{\nu}\right) \rightarrow \hat{K}_{n}^{\mathrm{et}}(A[1 / l]) .
\end{aligned}
$$

The first is an isomorphism by the finite generation of each $K_{n}(A)$ [31], the second an isomorphism by 8.1 , the third an epimorphism by 8.5 and finite generation, and the fourth an isomorphism by the finiteness of $H^{*}\left(A[1 / l]_{\mathrm{et}}, \mathbf{Z} / l^{\nu}(j)\right)$ for each $j$.

Since $K_{i}(A)$ is finite for $i>0$ if $A$ is the ring of $S$-integers in a function field [17, 18], the proof of 8.7 also gives the following result.

8.9 THEOREM. Let $A$ be a ring of $S$-integers in a function field of characteristic different from $l$. If $l=2$, assume $\sqrt{-1} \in A$. Then the natural map

$$
K_{n}(A) \rightarrow \hat{K}_{n}^{\mathrm{et}}(A) \approx H_{\text {cont }}^{j}\left(A_{\mathrm{et}}, \mathbf{Z}_{l}(i)\right)
$$

( $n=2 i-j, j=1,2)$ is surjective for $n \geqslant 1$.

The following divisibility theorem is a slightly sharpened version of the one announced in [7]. The theorem is stated for the ring of integers in a number field; there is an analogous result for rings of $S$-integers.

8.10 ThEOREM. Let $A$ be the ring of integers in a number field $F$. If $l=2$, assume $\sqrt{-1} \in A$. For any $j \geqslant 1$, there exists a finite Galois extension $F^{\prime}$ of $F$ with ring of integers $A^{\prime} \leqslant F^{\prime}$ such that

(i) $F^{\prime} / F$ is a solvable extension, unramified at any ( finite) prime not dividing $l$, and

(ii) the natural map

$$
K_{j}(A) / \text { torsion } \rightarrow K_{j}\left(A^{\prime}\right) / \text { torsion }
$$

has image divisible by $l$.

Proof. The groups $K_{2 i}(A)$ are finite for $i>0$ [2], so the theorem is nontrivial only for $j$ odd. Theorem 8.7 states that the natural map

$$
K_{2 i-1}(A) \otimes \mathbf{Z}_{l} \rightarrow \hat{K}_{2 i-1}^{\mathrm{et}}(A[1 / l]) \stackrel{\sim}{\rightarrow} H_{\mathrm{cont}}^{1}\left(A[1 / l]_{\mathrm{et}}, \mathbf{Z}_{l}(i)\right)
$$


is surjective for $i \geqslant 2$. Since the ranks of these groups have been computed and shown to be equal $[2,38]$ it follows that

$$
\left(K_{2 i-1}(A) / \text { torsion }\right) \otimes \mathbf{Z}_{l} \rightarrow \hat{K}_{2 i-1}^{\mathrm{et}}(A[1 / l]) / \text { torsion }
$$

is an isomorphism for $i \geqslant 2$. The case $j=1$ is easy to handle directly, so it suffices to exhibit $F \rightarrow F^{\prime}$ such that the image of $\hat{K}_{2 i-1}^{\text {et }}(A[1 / l]) \rightarrow K_{2 i-1}^{\text {et }}\left(A^{\prime}[1 / l]\right)$ is divisible by $l$. For this, it is enough to find $F \rightarrow F^{\prime}$ such that $K_{2 i-1}^{\text {et }}(A[1 / l], \mathbf{Z} / l) \rightarrow$ $K_{2 i-1}^{\mathrm{et}}\left(A^{\prime}[1 / l], \mathbf{Z} / l\right)$ is trivial, or, equivalently, such that $H^{1}\left(A[1 / l]_{\mathrm{et}}, \mathbf{Z} / l(i)\right) \rightarrow$ $H^{1}\left(A^{\prime}[1 / l]_{\mathrm{et}}, \mathbf{Z} / l(i)\right)$ is trivial. If $\zeta$ is a primitive $l$ th root of 1 , the extension $F[\zeta] / F$ is cyclic and unramified away from $l$, so it is no loss of generality to require $F=F[\zeta]$. Under this assumption, it is enough to construct $F \rightarrow F^{\prime}$ such that $H^{1}\left(A[1 / l]_{\mathrm{et}}, \mathbf{Z} / l(1)\right) \rightarrow H^{1}\left(A^{\prime}[1 / l]_{\mathrm{et}}, \mathbf{Z} / l(1)\right)$ is trivial. Consider the Kummer short exact sequence

$$
0 \rightarrow A[1 / l]^{*} \otimes \mathbf{Z} / l \rightarrow H^{1}(A[1 / l], \mathbf{Z} / l(1)) \rightarrow, \operatorname{Pic}(A[1 / l]) \rightarrow 0 .
$$

Let $F \rightarrow F^{\prime \prime}$ be the Hilbert class field extension, an unramified Galois extension with $\operatorname{Gal}\left(F^{\prime \prime} / F\right)=\operatorname{Pic}(A)$. It is known that $\operatorname{Pic}(A) \rightarrow \operatorname{Pic}\left(A^{\prime \prime}\right)$ is trivial, so that

$$
H^{1}\left(A[1 / l]_{\mathrm{et}}, \mathbf{Z} / l(1)\right) \rightarrow H^{1}\left(A^{\prime \prime}[1 / l]_{\mathrm{et}}, \mathbf{Z} / l(1)\right)
$$

has image contained in $A^{\prime \prime}[1 / l]^{*} \otimes \mathbf{Z} / l$. Let $F^{\prime \prime} \rightarrow F^{\prime}$ be the composite of the cyclic extensions unramified outside $l$ obtained by adjoining to $F^{\prime \prime}$ the $l$ th roots of enough units in $A^{\prime \prime}[1 / l]$ to generate $A^{\prime \prime}[1 / l]^{*} \otimes \mathbf{Z} / l$. Then $A^{\prime \prime}[1 / l]^{*} \otimes \mathbf{Z} / l \rightarrow$ $A^{\prime}[1 / l]^{*} \otimes \mathbf{Z} / l$ is trivial, so that $H^{1}\left(A[1 / l]_{\mathrm{et}}, \mathbf{Z} / l(1)\right) \rightarrow H^{1}\left(A^{\prime}[1 / l]_{\mathrm{et}}, \mathbf{Z} / l(1)\right)$ is trivial, as required.

Appendix: The space $\operatorname{Hom}_{g}(X, B G)_{S}$. The construction of the algebraic $K$-theory spectrum given in 4.1 involves geometric function spaces $\operatorname{Hom}_{g}(X, B G)_{S}$ (in the special case $S=\operatorname{Spec} R, G=\mathrm{GL}_{n}$ ). The purpose of this appendix is to calculate the homotopy type of such a function space. In the appendix, $G$ will denote a group scheme over an arbitrary (but fixed) base scheme $S$. If $U$ is a sscheme over $S$, then as usual $\operatorname{hom}(U, B G)_{S}$ will denote the set of maps from $U$ to $B G$ over $S$ and $\operatorname{Hom}(U, B G)_{S}$ the function space of maps from $U$ to $B G$ over $S$.

For any sscheme $U$ over $S$, let $Z^{1}(U, G)$ denote the set of all maps $f: U_{1} \rightarrow G$ (over $S$ ) which satisfy the (cocycle) condition

$$
\left(f \circ d_{2}\right) \cdot\left(f \circ d_{0}\right)=f \circ d_{1}: U_{2} \rightarrow G .
$$

A.1 Lemma. There is a natural isomorphism $\operatorname{hom}(U, B G)_{S} \rightarrow Z^{1}(U, G)$ given by $\left.f \rightarrow f\right|_{U_{1}}$.

Proof. This is an elementary consequence of the definition of $B G[\mathbf{1 4}, 1.2]$.

Let $\mathscr{H}^{1}(U, G)$ denote the category whose objects consist of the elements $f$ in $Z^{1}(U, G)$. A morphism $h: f \rightarrow f^{\prime}$ in this category is by definition a function $h$ : $U_{0} \rightarrow G$ (over $S$ ) such that $f \cdot\left(h \circ d_{0}\right)=\left(h \circ d_{1}\right) \cdot f^{\prime}: U_{1} \rightarrow G$. If $h$ is a morphism $f \rightarrow f^{\prime}$ and $h^{\prime}$ a morphism $f^{\prime} \rightarrow f^{\prime \prime}$, then $h \cdot h^{\prime}: U_{0} \rightarrow G$ represents the composite morphism $f \rightarrow f^{\prime \prime}$. 
A.2 Lemma. The set $Z^{1}(U \otimes \Delta[1], G)$ is naturally isomorphic to the set of arrows $h$ : $f_{0} \rightarrow f_{1}$ in the category $\mathscr{H}^{1}(U, G)$. More generally, the set $Z^{1}(U \otimes \Delta[n], G)$ is naturally isomorphic to the set of diagrams

$$
f_{0} \stackrel{h_{1}}{\rightarrow} f_{1} \stackrel{h_{2}}{\rightarrow} f_{2} \rightarrow \cdots \stackrel{h_{n}}{\rightarrow} f_{n}
$$

in $\mathscr{H}^{1}(U, G)$.

Proof. This follows from a direct calculation with the 2-skeleton of $U \otimes \Delta[n]$.

Lemmas A.1 and A.2 together imply

A.3 Lemma. The function space $\operatorname{Hom}(U, B G)_{S}$ is naturally isomorphic to the nerve of the category $\mathscr{H}^{1}(U, G)$.

Let $X$ be a scheme over $S$ and let $U \rightarrow X$ be an etale hypercovering. Denote by $T_{X}(G)$ the category of $G$-torseurs over $X$ and by $T_{X}(U, G)$ the full subcategory of $T_{X}(G)$ containing those $G$-torseurs $P$ which are $U_{0}$-trivial, i.e., $\left.P\right|_{U_{0}} \approx U_{0} \times G$.

A.4 Lemma. There is a natural functor $\mathscr{H}^{1}(U, G) \rightarrow T_{X}(U, G)$. This functor is an equivalence of categories.

Proof. There is one way to express the basic result of the theory of descent [6, I-4; 15, III.3.6].

In conjunction with A.3, Lemma A.4 leads to the following result.

A.5 Lemma. Let $X$ be a scheme over $S$ and let $U \rightarrow X$ be an etale hypercovering. Then there is a natural map from the simplicial set $\operatorname{Hom}(U, B G)_{S}$ to the nerve of the category $T_{X}(U, G)$. This map is a homotopy equivalence.

Taking a direct limit of the equivalences in A.5 provides an identification of $\operatorname{Hom}_{g}(X, B G)_{S}$.

A.6 TheOREM. Let $X$ be a scheme over $S$. For any left filtering category $I$ of etale hypercoverings of $X$ with the property that each $G$-torseur over $X$ is trivial over $U_{0}$ for some $U$ in $I$, there is a natural homotopy equivalence from $\lim _{U \in I} \operatorname{Hom}(U, B G)_{S}$ to the nerve of the category of $G$-torseurs over $X$.

Since the category of $G$-torseurs over $X$ is a groupoid category, its nerve contains as a strong deformation retract the disjoint union $\bigsqcup_{[P]} B \operatorname{Iso}(P)$, where $[P]$ runs through the set of isomorphism classes of $G$-torseurs over $X$, and $\operatorname{Iso}(P)$ denotes the group of automorphisms of the $G$-torseur $P$.

A.7 Corollary . Let $X$ be a scheme over $S$ and let $H^{1}(X, G)$ denote the set of isomorphism classes of $G$-torseurs over $X$. Then there is a homotopy equivalence

$$
\operatorname{Hom}_{g}(X, B G)_{S} \rightarrow \coprod_{[P] \in H^{1}(X, G)} B \operatorname{Iso}(P) .
$$




\section{REFERENCES}

1. S. Araki and H. Toda, Multiplicative structures on $\bmod q$ cohomology theories. I, Osaka J. Math. 2 (1965), 71-115.

2. A. Borel, Cohomology of arithemtic groups, Proc. 1974 Internat. Congress of Mathematicians, Vol. I, Canad. Math. Soc., 1975, pp. 435-442.

3. A. K. Bousfield and D. M. Kan, Homotopy limits, completions, and localizations, Lecture Notes in Math., vol. 304, Springer, New York, 1972.

4. __ Pairings and products in the homotopy spectral sequence, Trans. Amer. Math. Soc. 177 (1973), 319-343.

5. W. Browder, Algebraic K-theory with coefficients $Z / p$, Geometric Applications of Homotopy Theory, Lecture Notes in Math., vol. 657, Springer, New York, 1977, pp. 40-84.

6. P. Deligne, Cohomologie etale (SGA 4 1/2), Lecture Notes in Math., vol. 569, Springer, New York, 1977.

7. W. Dwyer and E. Friedlander, Etale K-theory and arithmetic, Bull. Amer. Math. Soc. (N.S.) 6 (1982), 453-455.

8. W. Dwyer, E. Friedlander, V. Snaith and R. Thomason, Algebraic K-theory eventually surjects onto topological K-theory, Invent. Math. 66 (1982), 481-491.

9. W. Dwyer and D. M. Kan, An obstruction theory for diagrams of simplicial sets (to appear).

10. E. Friedlander, Maps between localized homogeneous spaces, Topology 16 (1977), 205-216.

11. The infinite loop Adams conjecture via classification theorems for $\mathscr{F}$-spaces, Math. Proc. Cambridge Philos. Soc. 87 (1980), 109-150.

12. Etale K-theory I: Connections with etale cohomologv and algebraic vector bundles, Invent. Math. 60 (1980), 105-134.

13. Etale K-theory II: Connections with algebraic K-theory, Ann. Sci. École Norm. Sup. (4) 15 (1982), 231-256.

14. Et_ale homotopy of simplicial schemes, Ann. of Math. Stud., no. 104, Princeton Univ. Press, Princeton, N. J., 1982.

15. J. Giraud, Cohomologie non Abelienne, Springer, New York, 1971.

16. D. Grayson, Higher algebraic K-theory II (after D. Quillen), Algebraic K-theory, Lecture Notes in Math., vol. 551, Springer, New York, 1976, pp. 217-240.

17. Finite generation of $K$-groups of a curve over a finite field (after D. Quillen), Lecture Notes in Math., vol. 966, Springer, New York, 1982, pp. 69-90.

18. G. Harder, Die Kohomologie S-arithmetisches Gruppen über Funktionenkorpen, Invent. Math. 42 (1977), 135-175.

19. S. Lichtenbaum, Values of zeta functions, etale cohomology, and algebraic $K$-theory; classical algebraic K-theory and connections with arithmetic, Lecture Notes in Math., vol. 342, Springer, New York, 1973, pp. $489-501$.

20. J. L. Loday, K-theorie algebrique et representations de groupes, Ann. Sci. École Norm. Sup. (4) 9 (1976), 309-377.

21. S. Mac Lane, Categories for the working mathematician, Springer, New York, 1971.

22. J. P. May, Simplicial objects in algebraic topology, Van Nostrand, Princeton, N.J., 1967.

23.,$E_{\infty}$-spaces, group completions and permutative categories, New Developments in Topology,

London Math. Soc. Lecture Note Ser., Vol. 11, Cambridge University Press, Cambridge, 1974, pp. 61-93.

24. The spectra associated to permutative categories, Topology 17 (1978), 225-228.

25. __ Pairings of categories and spectra, J. Pure Appl. Algebra 19 (1980), 299-346.

26. D. McDuff and G. Segal, Homology fibrations and the "group completion" theorem, Invent. Math. 31 (1976), 279-284.

27. A. S. Merkurjev and A. A. Suslin, K-cohomology of Severi-Brauer varieties and the norm residue homomorphism, Izv. Akad. Nauk USSR (to appear).

28. J. S. Milne, Etale cohomology, Princeton Univ. Press, Princeton, N.J., 1980.

29. J. Milnor, Introduction to algebraic K-theory, Ann. of Math. Studies, no. 72, Princeton Univ. Press, Princeton, N.J., 1971.

30. D. G. Quillen, On the cohomologv and K-theory of the general linear group over finite fields, Ann. of Math. (2) 96 (1972), 552-586.

31. Finite generation of the groups $K_{i}$ of rings of algebraic integers, Lecture Notes in Math., vol. 341, Springer, New York, 1973, pp. 179-198. 
32. pp. $85-147$.

33. Higher algebraic K-theory, Proc. 1974 Internat. Congress of Mathematicians, Vol. I, Canad. Math. Soc., 1975, pp. 171-176.

34. G. Segal, Classifying spaces and spectral sequences, Inst. Hautes Études Sci. Publ. Math. 34 (1968), $105-112$.

35. Categories and cohomology theories, Topology 13 (1974), 293-312.

36. J. P. Serre, Cohomologie Galoisienne, Springer, New York, 1964.

37. C. Soulé, $K$-théorie des anneaux d'entièrs de corps de nombres et cohomologie étale, Invent. Math. 55 (1979), 251-295.

38. , On higher p-adic regulartors, Algebraic K-theory, Lecture Notes in Math., vol. 854, Springer, New York, 1981, pp. 372-401.

39. _ Operations on etale K-theory. Applications, Lecture Notes in Math., vol. 966, Springer, New York, 1982, pp. 271-303.

40. J. Tate, Relations between $K_{2}$ and Galois cohomologi, Invent. Math. 36 (1976), 257-274.

41. R. Thomason, The Lichtenbaum-Quillen conjecture for $K / I_{*}\left[\beta^{-1}\right]$, Current Trends in Algebraic Topology, Canadian Math. Soc. Conf. Proc., Vol. 2, Part I, Amer. Math. Soc., Providence, R.I., 1982, pp. $117-139$.

42. $87-109$. . Riemann-Roch for algebraic versus topological K-theory, J. Pure Appl. Algebra 27 (1983). 43. , Absolute cohomological purity, preprint, Johns Hopkins University.

Department of Mathematics, University of Notre Dame, Notre Dame, Indiana 46556

Mathematics Department, NorthWestern University, Evanston, IllinOIS 60201 